\title{
GRAVITATIONALLY UNSTABLE CONDENSATIONS REVEALED BY ALMA IN THE TUKH122 PRESTELLAR CORE IN THE ORION A CLOUD
}

\author{
Satoshi Ohashi ${ }^{1,2,3}$, Patricio Sanhueza ${ }^{2}$, Nami Sakai ${ }^{1}$, Ryo Kandori ${ }^{4}$, Minho Choi ${ }^{5}$, Tomoya Hirota $^{2,6}$, \\ QUANG NGUYêิN-LU'O'NG ${ }^{5}$, AND Ken'ICHi TATEMATSU ${ }^{2,6}$ \\ ${ }^{1}$ RIKEN, 2-1, Hirosawa, Wako-shi, Saitama 351-0198, Japan; satoshi.ohashi@riken.jp \\ 2 National Astronomical Observatory of Japan, 2-21-1 Osawa, Mitaka, Tokyo 181-8588, Japan \\ ${ }^{3}$ Department of Astronomy, Graduate School of Science, The University of Tokyo, 7-3-1 Hongo, Bunkyo-ku, Tokyo 113-0033, Japan \\ 4 Astrobiology Center of NINS, 2-21-1, Osawa, Mitaka, Tokyo 181-8588, Japan \\ ${ }^{5}$ Korea Astronomy and Space Science Institute, Daedeokdaero 776, Yuseong, Daejeon 305-348, South Korea and \\ ${ }^{6}$ Department of Astronomical Science, SOKENDAI (The Graduate University for Advanced Studies), 2-21-1 Osawa, Mitaka, Tokyo \\ 181-8588, Japan
}

\begin{abstract}
We have investigated the TUKH122 prestellar core in the Orion A cloud using ALMA 3 mm dust continuum, $\mathrm{N}_{2} \mathrm{H}^{+}(J=1-0)$, and $\mathrm{CH}_{3} \mathrm{OH}\left(J_{K}=2_{K}-1_{K}\right)$ molecular line observations. Previous studies showed that TUKH122 is likely on the verge of star formation because the turbulence is almost dissipated and chemically evolved among other starless cores in the Orion A cloud. By combining ALMA 12-m and ACA data, we recover extended emission with a resolution of $\sim 5^{\prime \prime}$ corresponding to $0.01 \mathrm{pc}$ and identify 6 condensations with a mass range of $0.1-0.4 M_{\odot}$ and a radius of $\lesssim 0.01$ pc. These condensations are gravitationally bound following a virial analysis and are embedded in the filament including the elongated core with a mass of $\sim 29 M_{\odot}$ and a radial density profile of $r^{-1.6}$ derived by Herschel. The separation of these condensations is $\sim 0.035 \mathrm{pc}$, consistent with the thermal jeans length at a density of $4.4 \times 10^{5} \mathrm{~cm}^{-3}$. This density is similar to the central part of the core. We also find a tendency that the $\mathrm{N}_{2} \mathrm{H}^{+}$molecule seems to deplete at the dust peak condensation. This condensation may be beginning to collapse because the linewidth becomes broader. Therefore, the fragmentation still occurs in the prestellar core by thermal Jeans instability and multiple stars are formed within the TUKH122 prestellar core. The $\mathrm{CH}_{3} \mathrm{OH}$ emission shows a large shell-like distribution and surrounds these condensations, suggesting that the $\mathrm{CH}_{3} \mathrm{OH}$ molecule formed on dust grains is released into gas phase by non-thermal desorption such as photoevaporation caused by cosmic-ray induced UV radiation.

Keywords: ISM: clouds -stars: formation -ISM: individual (Orion Molecular Cloud) - ISM: molecules
\end{abstract}

\section{INTRODUCTION}

To understand star formation processes, it is of great importance to reveal the initial conditions of star formation. In nearby dark clouds at a distance of $\sim 100 \mathrm{pc}$, many observations have been performed using molecular lines and continuum emission and identified dense cores (or molecular dense cores) as birth places of stars (e.g., Myers \& Benson 1983; Benson \& Myers 1989; WardThompson et al. 1994; Onishi et al. 2002; Caselli et al. 2002). One of the most important information to understand the dense core properties is the density structure. On the basis of near-infrared observations, Alves et al. (2001) showed that the Bonnor-Ebert Sphere model (Bonnor 1956; Ebert 1955) can explain the observed radial column density profile of the Barnard 68. Many nearby dense cores have also shown the Bonnor-Ebert structure (Kandori et al. 2005). The Bonnor-Ebert shape consists of a flat central region surrounded by a steeper outer region of $\rho \propto r^{-2}$. Such a simple dense core is suggested to form a single protostar, binary stars, and multiple stars by gravitational collapse (e.g., Matsumoto \& Hanawa 2003).

The dense cores may be the most simplified description to determine the star-forming process and is often assumed as the initial conditions for simulations. Therefore, the simple density structure can be applied to these isolated objects except for multiple star systems (e.g.,
Tokuda et al. 2014, 2016; Dunham et al. 2016; Kirk et al. 2017). Recently, Pineda et al. (2010, 2011, 2015) have discovered the quiescent thermal dense core, B5 in Perseus, showing a quadruple star system inside the core with the $\mathrm{NH}_{3}$ high resolution observations. They suggested that the multiple star system is formed because the coherent core is fragmented into dense filaments with length of $5000 \mathrm{au}$. Taking into account these results, the fragmentation in prestellar stage of dense cores may be needed to be a multiple star system. Therefore, it is important to reveal the smallest and densest parts of the dense cores where stars are born.

However, the properties of dense cores in various starforming environments have not been fully explored yet. The majority of stars are thought to be produced through the formation of clusters (Lada \& Lada 2003). In particular, "giant molecular clouds (GMCs)" are well known to be major sites of star formation in our Galaxy, and often show star cluster formation including massive stars. Therefore, it is essential to observe the dense cores embedded in GMCs to reveal the initial conditions of star formation. Furthermore, the observational targets should be dense cores on the verge of star formation.

To search for dense cores with the initial conditions of star formation in GMCs, chemical evolution may be one of the powerful tools to determine the evolutionary stages of the dense cores. A pioneering study on the 
chemical evolution in dark clouds was done by Suzuki (1983) and Suzuki et al. (1992). They suggested that carbon chain molecules such as $\mathrm{CCS}, \mathrm{HC}_{3} \mathrm{~N}$, and $\mathrm{HC}_{5} \mathrm{~N}$ are abundant in dense cores in the early stages of star formation because reactions involving atomic carbon or ionized carbon are effective in early stage and they are easily depleted onto dust in later stage (Aikawa et al. 2001). They also suggested that the N-bearing molecule $\mathrm{NH}_{3}$ is abundant in dense cores in the later stage because $\mathrm{N}_{2}$ molecule (precursor to N-bearing molecules) is slowly formed. Tatematsu et al. (1993, 2014a) showed that $N\left(\mathrm{~N}_{2} \mathrm{H}^{+}\right) / N(\mathrm{CCS})$ may indicate the chemical evolutionary stage. Similarly, Ohashi et al. (2014) found that the $\mathrm{NH}_{3} / \mathrm{CCS}$ column density ratio is anti-correlated with the CCS linewidth, and suggested that chemical evolution and turbulence dissipation can be indicators of the dynamical evolution of cores. Therefore, if we can identify dense cores that are rich in N-bearing molecules and poor in carbon-chain molecules without protostars, these cores will tell us the initial conditions of star formation.

Based on these studies, such cores are searched for in the Orion A cloud, the Vela C molecular cloud complex, and Planck cold clumps (Ohashi et al. 2014, 2016a; Tatematsu et al. 2014a, 2017). In particular, the Orion A cloud is a good target because it is one of the nearest GMCs from the earth and has been well studied. Tatematsu et al. (2014a) have found a dense core (TUKH122) having the largest value of $N\left(\mathrm{~N}_{2} \mathrm{H}^{+}\right) / N(\mathrm{CCS})$ among the starless dense cores in the Orion A cloud. TUKH122 has $N\left(\mathrm{~N}_{2} \mathrm{H}^{+}\right) / N(\mathrm{CCS}) \sim 3$ (typical starless dense cores have $\sim 1.5)$ and is located in the L1641 South region (see Figure 1). TUKH122 could be the dense core closest to star formation in our samples. Note that no IRAC, MIPS, or SDSS sources are detected toward TUKH122, suggesting that it is in the prestellar phase.

Detailed observations have been performed toward TUKH122 with the VLA and Nobeyama $45 \mathrm{~m}$ telescope. The VLA $\mathrm{NH}_{3}$ observations have identified an oval core with a size of $0.1 \mathrm{pc}$ and condensations with a size of 0.03 pc embedded in the parent CS clump. The linewidth of $\mathrm{NH}_{3}$ is narrow $\left(\sim 0.2 \mathrm{~km} \mathrm{~s}^{-1}\right)$, and both the core and condensation are gravitationally bound (Tatematsu et al. 2014b). The single pointing $\mathrm{NH}_{3}$ observations covering the whole main core with $0.05 \mathrm{~km} \mathrm{~s}^{-1}$ velocity resolution identified not only the thermal narrow component but also a turbulent component, suggesting that the sharp transition from the parent clump to the quiescent dense core (Ohashi et al. 2016b). These results suggested that the TUKH122 core is on the verge of star formation and is one of the good targets to investigate the physical conditions and fragmentation process if this core forms multiple stars. However, these observations were not enough to reveal the density distribution or fragmentation due to the resolution and sensitivity.

Recently, the Herschel Space Observatory has revealed that filaments are ubiquitous in star-forming clouds and dense cores are embedded in the filaments (e.g., André et al. 2010; Arzoumanian et al. 2011; Hill et al. 2011). Therefore, TUKH122 is also important to understand dense core formation in the filaments in GMCs.

In this paper, we report ALMA observations toward TUKH122 at Band 3 with $\sim 5^{\prime \prime}$ beam. Note that we will follow the nomenclature of Ohashi et al. (2016c) and
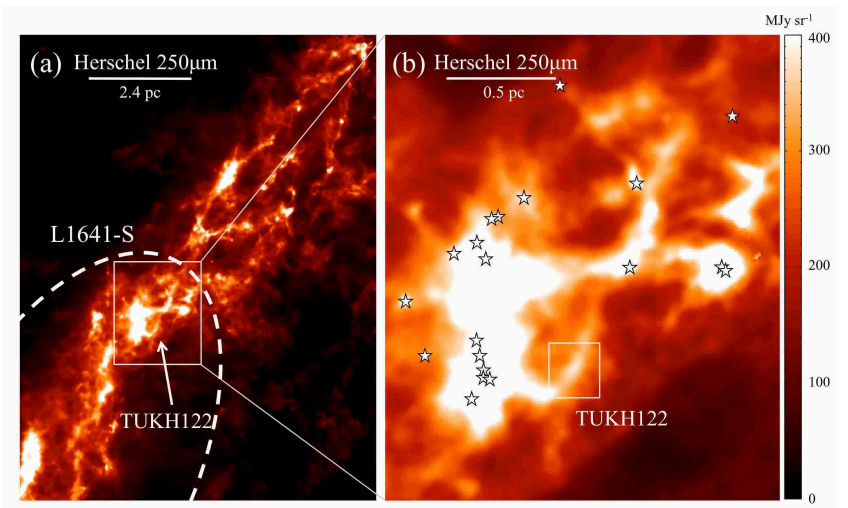

Figure 1. The Herschel $250 \mu \mathrm{m}$ images. The left panel (a) shows a wide field and indicates L1641-S region. The right panel (b) shows the zoom up toward TUKH122. The open stars represent the locations of protostars identified by Spitzer (Megeath et al. 2012).

refer to cores as an entity of $0.01-0.1 \mathrm{pc}$. We also refer to a dense condensation as an entity of $\lesssim 0.01 \mathrm{pc}$. The distance of Orion A cloud is derived to be 418 pc (Kim et al. 2008).

In Section 2, we describe the observations and data reduction. In Section 3, we present and describe the dust continuum maps taken by the Herschel, ACA, and ALMA observations as well as the molecular line maps of the ACA and ALMA data. In Section 4, we discuss the formation processes of condensations in the core and possible scenario of multiple star systems. Our conclusions are summarized in Section 5.

\section{OBSERVATIONS}

TUKH122 was observed with the ALMA 12-m Array on 2016 March 10-12 in the C36-2/3 configuration with a total of 38 antennas and with the 7-m Array of the Atacama Compact Array (ACA; also known as the Morita Array; Iguchi et al. 2009) on 2016 May 28, August 27, and September 1-7 with a total of 10 antennas (Cycle 3 program, Project ID: 2015.1.01025.S). The number of $12-\mathrm{m}$ pointings for the mosaic mapping is five. The Band 3 receivers were used. The system temperature was in the range of 40 to $140 \mathrm{~K}$. The correlator was set to have four spectral windows (two windows for continuum, one for $\mathrm{N}_{2} \mathrm{H}^{+}$, and one for $\mathrm{CH}_{3} \mathrm{OH}$ ). The velocity resolution of the molecular line observations was set to be $\sim 0.098 \mathrm{~km} \mathrm{~s}^{-1}$. The four quasars (J0423-0120, J05223627 , J0854+2006, and J0510+1800) were observed for bandpass calibration. J0542-0913 was observed for phase calibration. Flux calibration was performed using J04230120, J0510+1800, J0522-3627, and Uranus.

The reduction and calibration of the data were done with CASA version 4.5.3 (McMullin et al. 2007) in a standard manner. All images were reconstructed with the CASA task CLEAN using natural weighting. To improve the sensitivity, we also used uv tapering $(50 \mathrm{k} \lambda$ for the molecular lines and $20 \mathrm{k} \lambda$ for continuum) for CLEAN. The pixel size was set to $00^{\prime \prime} 5$. To make high spatial resolution images recovering the extended emission, we combine the ALMA 12-m and ACA in the uv plane. The resultant spatial resolutions are written in each section. 
(a) Herschel $250 \mu \mathrm{m}$

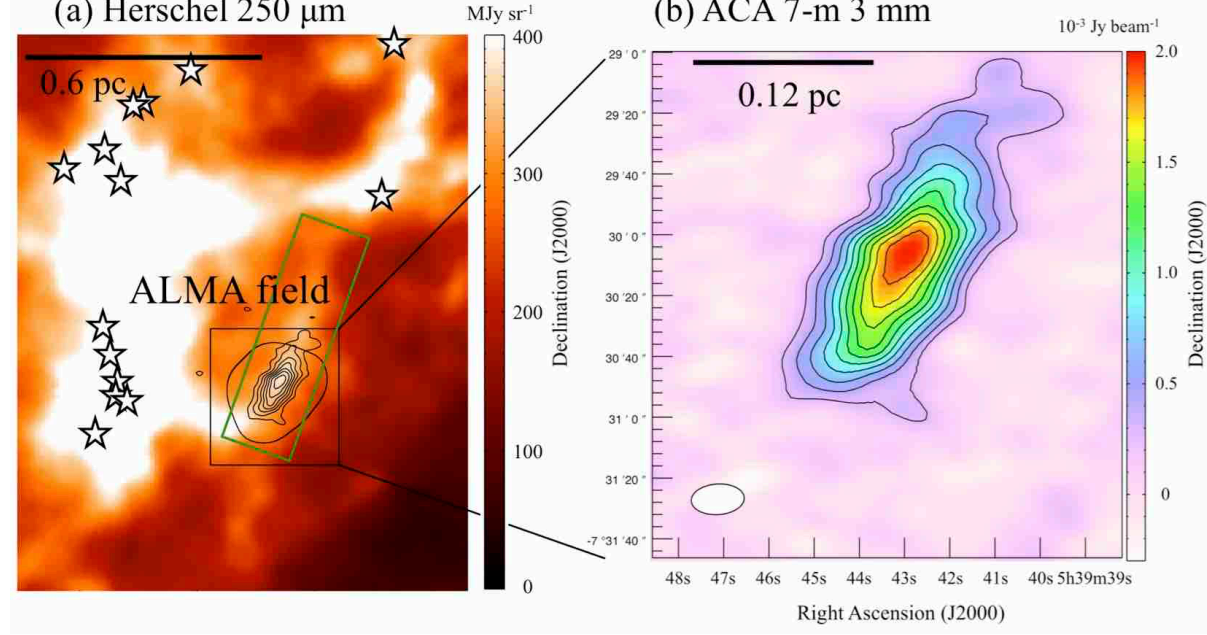

(c) ALMA 12-m $3 \mathrm{~mm}$

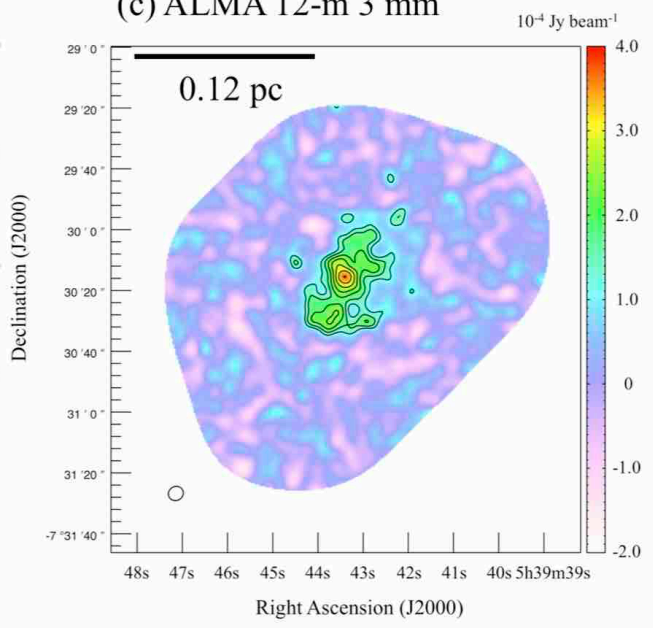

Figure 2. (a) The Hershcel $250 \mu \mathrm{m}$ dust continuum image toward the southern part of the Orion A cloud. The black contour shows the ALMA 12-m observed field toward TUKH122. The star symbols represent the protostars identified by Spitzer. The contours show the ACA $3 \mathrm{~mm}$ dust continuum emission as the same with panel (b). The green box shows the parent filamentary region. (b) The ACA 7-m $3 \mathrm{~mm}$ dust continuum image without the primary beam correction toward TUKH122 core. The contours start at $3 \sigma$ with intervals of $3 \sigma$. The $1 \sigma$ noise level is $87 \mu \mathrm{Jy}$ beam ${ }^{-1}$. The bottom left circle represents the beamsize of $17^{\prime \prime} \times 10^{\prime \prime}\left(\mathrm{PA}=-86^{\circ}\right)$ corresponding to 0.035 pc $\times 0.02$ pc. (c) The $3 \mathrm{~mm}$ dust continuum image without the primary beam correction toward TUKH122 core obtained by the ALMA 12 -m array. The contours start at $3 \sigma$ with intervals of $1 \sigma$. The $1 \sigma$ noise level is $43 \mu \mathrm{Jy}_{\text {beam }}{ }^{-1}$. The bottom left circle represents the beamsize of 5.' $0 \times 4 .^{\prime \prime} 6\left(\mathrm{PA}=-61^{\circ}\right)$ corresponding to $0.01 \mathrm{pc} \times 0.0094 \mathrm{pc}$. The combined ALMA image is shown in Figure 4 .

Table 1

Beam Sizes and Sensitivities

\begin{tabular}{lllc}
\hline \hline Parameters & ALMA $12 \mathrm{~m}$ Array & ACA $7 \mathrm{~m}$ Array & ALMA-ACA combined \\
\hline Synthesized beam size of continuum observation & $\sim 5^{\prime \prime} 0 \times 4^{\prime \prime} \cdot 6$ & $\sim 17^{\prime \prime} \times 10^{\prime \prime}$ & $\sim 5^{\prime \prime} 7 \times 5^{\prime \prime} 2$ \\
Synthesized beam size of line observation & $\sim 3.2^{\prime \prime} 0 \times 2^{\prime \prime} 7$ & $\sim 18^{\prime \prime} \times 10^{\prime \prime}$ & $\sim 3^{\prime \prime} 2 \times 2^{\prime \prime} 9$ \\
Sensitivity of continuum observation $(\mathrm{rms})$ & $\sim 43 \mu \mathrm{Jy} \mathrm{beam}^{-1}$ & $\sim 87 \mu \mathrm{Jy} \mathrm{beam}^{-1}$ & $\sim 40 \mu \mathrm{Jy} \mathrm{beam}^{-1}$ \\
Sensitivity of line observation $(\mathrm{rms})^{a}$ & $\sim 6.5$ mJy beam $^{-1}$ & $\sim 31 \mathrm{mJy} \mathrm{beam}^{-1}$ & $\sim 6.5 \mathrm{mJy} \mathrm{beam}^{-1}$ \\
\hline
\end{tabular}

a The velocity resolution is $\sim 0.098 \mathrm{~km} \mathrm{~s}^{-1}$

The synthesized beams and the sensitivities of the dust continuum and the molecular lines are listed in Table 1.

\section{RESULTS}

Figure 2 shows the dust continuum maps toward the TUKH122 region at different scales with Herschel and ALMA. The various observations with the different spatial resolutions reveal the different structures from filament to condensation. Figure 2 (a) shows the location of TUKH122 on the Herschel $250 \mu \mathrm{m}$ map (Gould Belt Survey Archive: André et al. 2010; Roy et al. 2013; Polychroni et al. 2013) overlaid with ACA 3 mm dust continuum emission contours. We find a filamentary structure along north-south direction with a length of $\sim 0.6 \mathrm{pc}$ shown in the green box, which is wider than ALMA observed field.

Figures 2 (b) and (c) show the $3 \mathrm{~mm}$ dust continuum images obtained by ACA 7-m array and ALMA 12-m, respectively. The beamsize is $17^{\prime \prime} \times 10^{\prime \prime}\left(\mathrm{PA}=-86^{\circ}\right)$ for ACA and 5.! $0 \times 4$ !. $6^{\prime \prime}\left(\mathrm{PA}=-61^{\circ}\right)$ for ALMA $12-\mathrm{m}$. These images are not corrected by the primary beam pattern to keep the same noise level within the images. The ACA observations show the simple elongated structure $(\sim 0.12$ $\mathrm{pc} \times 0.048 \mathrm{pc}$ in $\mathrm{FWHM})$ toward TUKH122 region. In the 12-m array data (Figure $2 \mathrm{c}$ ), compact condensations are clearly seen, whereas such components are not spatially resolved in the $7-\mathrm{m}$ array data. The ALMA-12m observations only reveal the small condensations within the core.

The oval structure of the TUKH122 core was already reported by Tatematsu et al. (2014b) with VLA NH 3 observations. Figure 3 shows the ACA $7-\mathrm{m} 3 \mathrm{~mm}$ dust continuum image with the $\mathrm{VLA} \mathrm{NH}_{3}$ integrated intensity contours. The $3 \mathrm{~mm}$ dust continuum emission taken by ACA is extended compared with the $\mathrm{VLA} \mathrm{NH}_{3}$ emission, which may be explained by filtering out in the VLA interferometric data. These oval structures are consistent with the typical dense core found in the low-mass star forming region.

We suggest that the TUKH122 core is embedded in a parent filament having larger scale emission. However, it is difficult to distinguish the core emission component from the filament. Therefore, the Herschel and ACA observations trace the combination of the filament and the core components. Most emission may be from the core because the TUKH122 core has been already formed.

Figure 4 shows the $3 \mathrm{~mm}$ dust continuum image obtained by the ALMA-ACA combined data. The contours start at $3 \sigma$ with intervals of $1 \sigma$. The $1 \sigma$ noise level is 40 $\mu \mathrm{Jy}$ beam $^{-1}$ and the beamsize is $5^{\prime \prime} 7 \times 5^{\prime \prime} .2\left(\mathrm{PA}=-66^{\circ}\right)$.

\subsection{Analysis of the Herschel and ALMA continuum data}




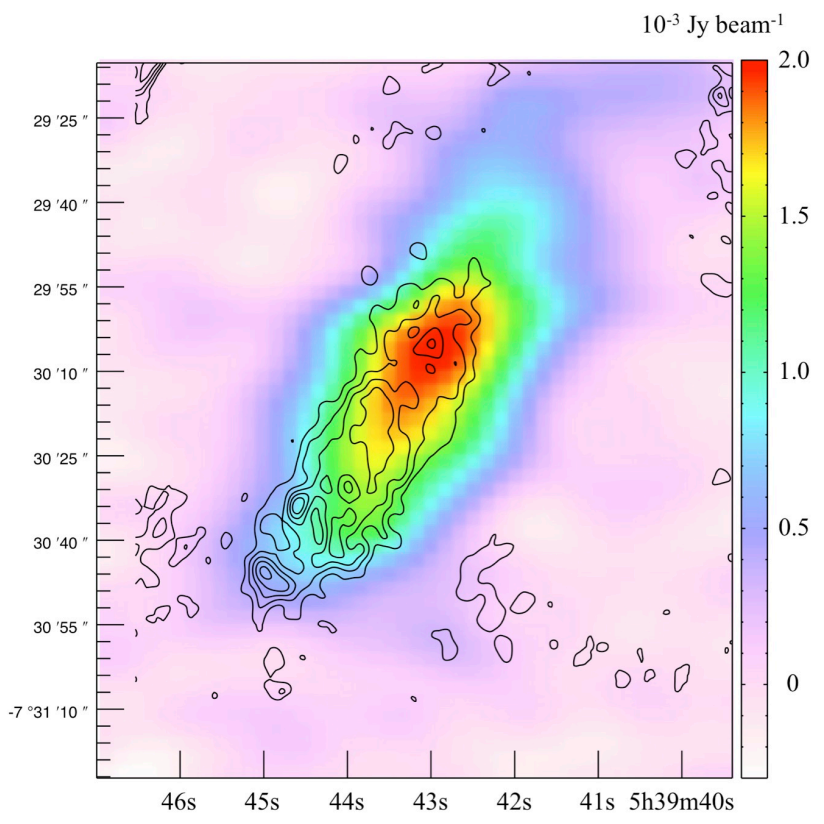

Figure 3. The color map shows ACA $7-\mathrm{m} 3 \mathrm{~mm}$ dust continuum image without the primary beam correction toward TUKH122 core. The contours show $\mathrm{NH}_{3}$ integrated intensity map. The lowest contour level and contour interval are $2.4 \mathrm{mJy} \mathrm{beam}^{-1} \mathrm{~km} \mathrm{~s}^{-1}$.

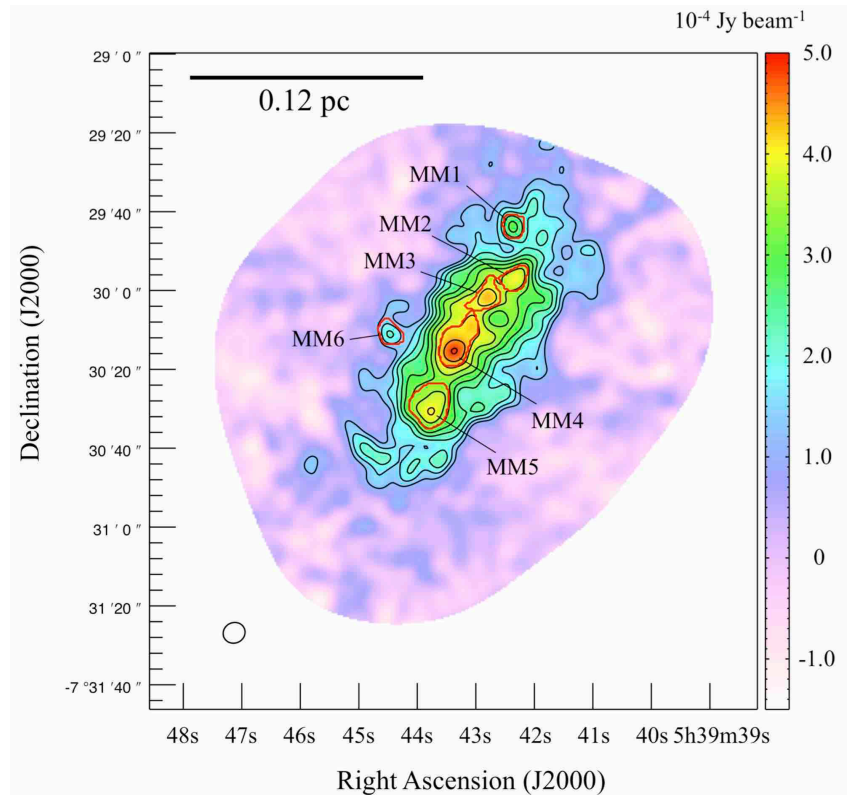

Figure 4. The ALMA-ACA combined $3 \mathrm{~mm}$ dust continuum image toward TUKH122 core without the primary beam correction. The contours start at $3 \sigma$ with intervals of $1 \sigma$. The $1 \sigma$ noise level is $40 \mu \mathrm{Jy} \mathrm{beam}^{-1}$. The red contours represent the condensations. The bottom left circle represents the beamsize of 5. . $7 \times 55^{\prime \prime} 2$ $\left(\mathrm{PA}=-66^{\circ}\right)$.

To derive the dust temperature and mass of the ALMA-ACA observing region by using the Hershcel 250, 350 , and $500 \mu \mathrm{m}$ data, we adopt a dust opacity of $5.5 \mathrm{~cm}^{2}$ $\mathrm{g}^{-1}$ at $350 \mu \mathrm{m}$ and $2.7 \mathrm{~cm}^{2} \mathrm{~g}^{-1}$ at $500 \mu \mathrm{m}$ modeled by Ormel et al. (2007). These values have been shown to reporduce well the observed SED from 2.2 to $850 \mu \mathrm{m}$ in the Orion A cloud as suggested by Lombardi et al. (2014).
The mass $M$, and dust temperature $T_{d}$, are computed with

$$
M=\frac{F_{\nu} d^{2}}{\kappa B_{\nu}\left(T_{d}\right)} f_{d},
$$

where $F_{\nu}$ is the observed flux in $\mathrm{Jy}, d$ is the distance to the target, $B_{\nu}\left(T_{d}\right)$ is the Planck function, $\kappa$ is the dust opacity, and $f_{d}$ is the gas to dust mass ratio (assumed to be 100). By calculating the flux density within the ALMA observed field, the dust temperature of TUKH122 is derived to be $12 \mathrm{~K}$, consistent with the previous results of $T_{\text {kin }}=11 \mathrm{~K}$ obtained by $\mathrm{NH}_{3}$ spectra (Ohashi et al. $2016 \mathrm{~b})$. The mass is also derived to be $\sim 29 M_{\odot}$. In spite of the observational studies that dust temperature is often higher than gas temperatures because of averaging the line-of-sight of the dust, the similar temperatures between gas and dust may suggest that this region is widely isothermal.

The plane of the sky orientation of the filament and elongated core is measured to be a position angle of P.A. $=146.3$ degrees derived by $2 \mathrm{D}$ gaussian fitting on the Herschel image. The directions of the parallel and perpendicular to the filamentary structure are shown in Figure 5.

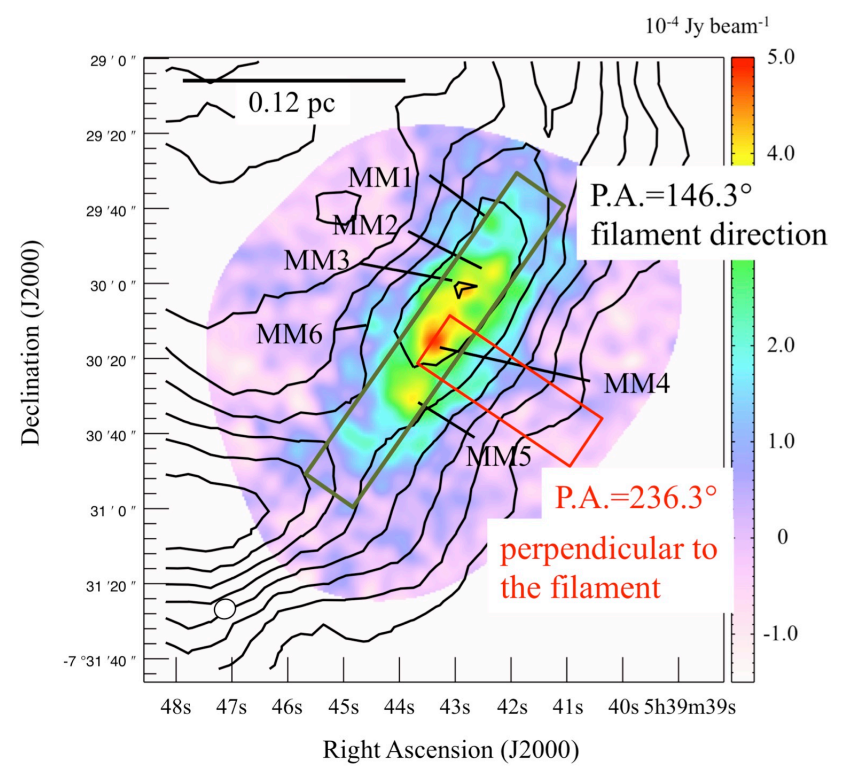

Figure 5. The color map shows the same as Figure 4. The contours show the Herschel $250 \mu \mathrm{m}$ dust continuum and start at 260 $\mathrm{MJy} \mathrm{sr}^{-1}$ with $20 \mathrm{MJy} \mathrm{sr}^{-1}$ intervals. The directions of the parallel and perpendicular to the filamentary structure are also shown with a width of $15^{\prime \prime}$.

To investigate the density structure of this region, Figure 6 shows the column density profiles perpendicular to the filamentary structure. The column density profiles are derived with the ALMA-ACA and Herschel observations by using the equation (1) assuming the surface are of the ALMA-ACA beamsize of $\sim 5$.' 2 and the Herschel beamsize of $\sim 18^{\prime \prime}$. We measure a radial column density profile to the south-westen direction $\left(\right.$ P.A. $\left.=236.3^{\circ}\right)$ perpendicular to the filament. Then, the profile is calculated by averaging the flux densities within $15^{\prime \prime}$ width at each distance from the peak position (see also Figure 5). We 
do not use the data of the north-eastern side because several protostars may affect the density and temperature in this side (see Figure 2).

Figure 6 shows that the Herschel observations recover extended emission at a distance larger than $0.1 \mathrm{pc}$, while ALMA-ACA observations only detect dense gas within $0.1 \mathrm{pc}$. This is consistent with the results that the ALMA 12-m and ACA 7-m observations are not sensitive to the extended envelope for scale larger than $0.1 \mathrm{pc}$. We also find that the column densities of the ALMA-ACA observations are higher than that of the Herschel observations inside $0.02 \mathrm{pc}$. This would most likely be caused by the low resolution of the Herschel observation because the ALMA-ACA observations are three times better spatial resolution than Herschel. For example, Herschel does not resolve the $0.04 \mathrm{pc}$ scale condensation found in this study.

To understand the density structure of the parent filament with the core, we plot the Plummer like function as the red and black lines in the figure. The Plummer-like profile is defined as

$$
\rho(r)=\frac{\rho_{c}}{\left[1+\left(r / R_{f}\right)^{2}\right]^{p / 2}},
$$

and the corresponding surface density profile is

$$
\Sigma(r)=A \frac{\rho_{c} R_{f}}{\left[1+\left(r / R_{f}\right)^{2}\right]^{(p-1) / 2}},
$$

where $A$ is a numerical factor. The numerical factor of $A$ depends on the density slope $p . A=\pi$ corresponds to be $p=2$, while $A=\pi / 2$ corresponds to be $p=4$ (Arzoumanian et al. 2011). We derive the column density assuming the mean molecular weight of 2.33 . The profile is determined by the central density $\rho_{c}$, the radius $R_{f}$, and the density slope $p$ (Nutter et al. 2008; Arzoumanian et al. 2011). Note that the isothermal hydrostatic filament has $p=4$ (Stodólkiewicz 1963; Ostriker 1964). Recently the Herschel observations suggest $p \sim 2$ (e.g., Arzoumanian et al. 2011) and that the dynamical contraction makes a flatter density slope. In Figure 6, the red line shows the power law of $p=1.6$, while the black solid line shows the power law of $p=4.0$, nicely fitting the data plotted. The FWHM of the Herschel data fitting is $0.16 \mathrm{pc}$. On the other hand, the ALMA-ACA observations show a thinner and steeper filament due to the missing flux.

The slope of $p=4.0$ is consistent with the isothermal hydrostatic filament or the density profile of prestellar cores (Whitworth \& Ward-Thompson 2001). However, the ALMA-ACA observations only recover 8.4 $M_{\odot}$ out of a total of $\sim 29 M_{\odot}$. It is difficult to distinguish the density structure of the TUKH122 core from the parent filament by these observations due to the missing flux. We cannot discuss the density profile or the power law of the ALMA-ACA observations. We only show that the missing flux is not the background emission but the surrounding gas associated to the filament.

It is possible that the power law of $p=1.6$ indicates the dynamical contraction of the filament rather than the background effect. The filament actually forms the dense core and condensations. Therefore, we indicate that the power law of $p=1.6$ is the radial profile of the parent filament that already forms the elongated core.

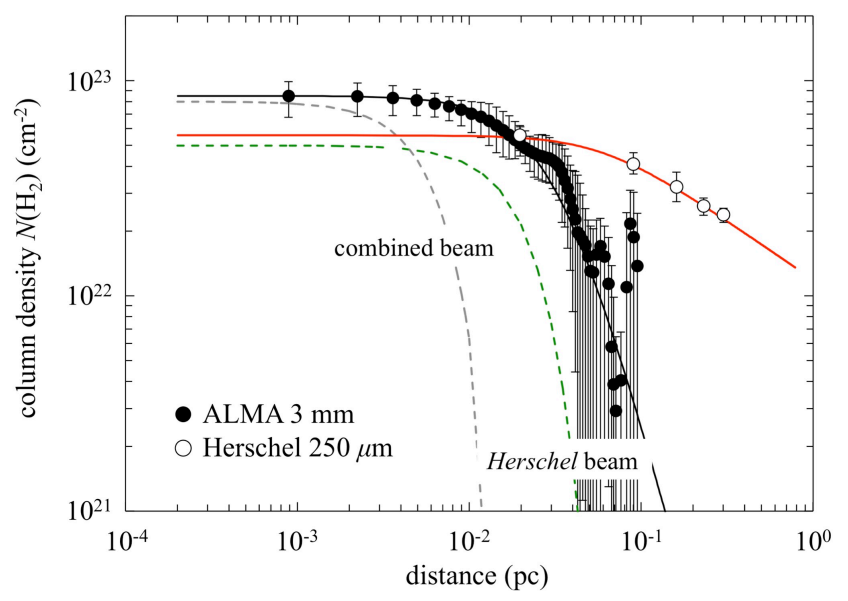

Figure 6. The column density profiles perpendicular to the filament direction taken by ALMA-ACA observations and the Herschel $250 \mu \mathrm{m}$ observations. Error bars show the maximum and minimum distributions of the profiles in each region. The black and red lines represent the Plummer like function with the power laws of 4.0 and 1.6, respectively. The dash lines represents the beam patterns of the ALMA-ACA combined data and Herschel $250 \mu \mathrm{m}$ data.

\subsection{The $3 \mathrm{~mm}$ dust continuum emission seen by ALMA}

To identify dense condensations precisely, we apply the dendrogram method (Rosolowsky et al. 2008) to the combined $3 \mathrm{~mm}$ dust continuum image. The dendrogram traces local maxima and describes hierarchical structures, which have been shown to be more robust against noise and user defined parameters (e.g., Goodman et al. 2009; Pineda et al. 2009). This method gives three types of structures: leaf, branch, and trunk. We define "leaves" as "condensations" in this paper because the leaf is the smallest structure. We adopt a threshold of $3 \sigma$ and $1 \sigma$ interval steps. The minimum number of pixels are $3 \times \theta_{\text {beam }}$. In addition, we select the condensations as the robust one when they can be identified as detection above $3 \sigma$ with ALMA $12-\mathrm{m}$ data. The identified condensations are shown in Figure 4 as the red contours and listed in Table 2.

The mass of the $3 \mathrm{~mm}$ dust condensations are computed with the equation (1) at a dust temperature $T_{d}=$ $12 \mathrm{~K}$. We adopt a dust opacity of $0.0755 \mathrm{~cm}^{2} \mathrm{~g}^{-1}$, assuming a dust emissivity index of $\beta=2$ (Ormel et al. 2007). As a result, the condensation mass ranges from 0.1 to $0.4 M_{\odot}$. The typical deconvolved radius is $r \sim 2^{\prime \prime} \cdot 9$. The radius is calculated from the surface area of the identified condensations. The $\mathrm{H}_{2}$ densities are $n \sim 10^{6-7}$ $\mathrm{cm}^{-3}$ which are of the order of or higher than those of typical low-mass prestellar cores (e.g., Onishi et al. 2002; Enoch et al. 2008). The condensation in the center of TUKH122, MM4, is the most massive and has $\sim 0.4$ $M_{\odot}$. Note that the deconvolved radius of $r \sim 22^{\prime \prime} 9$ is very close to the beamsize radius. The condensations MM1,2 and 6 show that the peak intensities have larger values than the total fluxes (see also Table 2). These results indicate that these three condensations (MM1,2 and 6) are not well resolved with our observations. However, the distances between the condensations are larger than the beamsize, indicating that we can determine the locations of the condensations with our observations. We find an 
Table 2

Physical Parameters of dense condensations

\begin{tabular}{|c|c|c|c|c|c|c|c|c|c|c|}
\hline Sources & $\begin{array}{l}\text { R.A. } \\
\text { (h:m:s) }\end{array}$ & $\begin{array}{c}\text { Decl } \\
\text { (d:m:s) }\end{array}$ & $\begin{array}{c}S_{\text {peak }}^{\mathrm{a}} \\
\left(\mathrm{mJy} \mathrm{beam}^{-1}\right)\end{array}$ & $\begin{array}{l}S_{\text {int }}^{\mathrm{a}} \\
(\mathrm{Jy})\end{array}$ & $\begin{array}{l}\text { Mass }^{\mathrm{b}} \\
\left(M_{\odot}\right)\end{array}$ & $\begin{array}{l}r^{\mathrm{c}} \\
\left({ }^{\prime \prime}\right)\end{array}$ & $\begin{array}{c}n \\
\left(\mathrm{~cm}^{-3}\right)\end{array}$ & $\begin{array}{l}\text { Velocity }^{\mathrm{d}} \\
\left(\mathrm{km} \mathrm{s}^{-1}\right)\end{array}$ & $\begin{array}{c}\text { linewidth }^{\mathrm{d}} \\
\left(\mathrm{km} \mathrm{s}^{-1}\right)\end{array}$ & $\begin{array}{c}\text { Virial Mass } \\
\left(M_{\odot}\right) \\
\end{array}$ \\
\hline MM-1 & 5:39:42.34 & $-7: 29: 43$ & 0.45 & 0.33 & 0.12 & 1.6 & $1.5 \times 10^{7}$ & 3.81 & 0.20 & 0.03 \\
\hline MM-2 & $5: 39: 42.30$ & $-7: 29: 56$ & 0.46 & 0.43 & 0.15 & 2.0 & $9.2 \times 10^{6}$ & 3.76 & 0.22 & 0.04 \\
\hline MM-3 & $5: 39: 42.78$ & $-7: 30: 01$ & 0.46 & 0.55 & 0.20 & 2.7 & $5.2 \times 10^{6}$ & 3.80 & 0.20 & 0.05 \\
\hline MM-4 & $5: 39: 43.37$ & $-7: 30: 15$ & 0.48 & 1.1 & 0.39 & 4.5 & $2.2 \times 10^{6}$ & 3.77 & 0.38 & 0.3 \\
\hline MM-5 & $5: 39: 43.76$ & $-7: 30: 31$ & 0.42 & 0.99 & 0.36 & 4.7 & $1.8 \times 10^{6}$ & 3.79 & 0.22 & 0.1 \\
\hline MM-6 & $5: 39: 44.48$ & $-7: 30: 10$ & 0.22 & 0.17 & 0.06 & 1.9 & $4.9 \times 10^{6}$ & 3.66 & 0.23 & 0.04 \\
\hline
\end{tabular}

${ }^{a}$ Fluxes are corrected for the primary beam attenuation.

b The mass is derived from the combined map.

c The deconvolved size of the radius.

d The velocity and linewidth are derived by Gaussian fitting of $\mathrm{CH}_{3} \mathrm{OH}$.
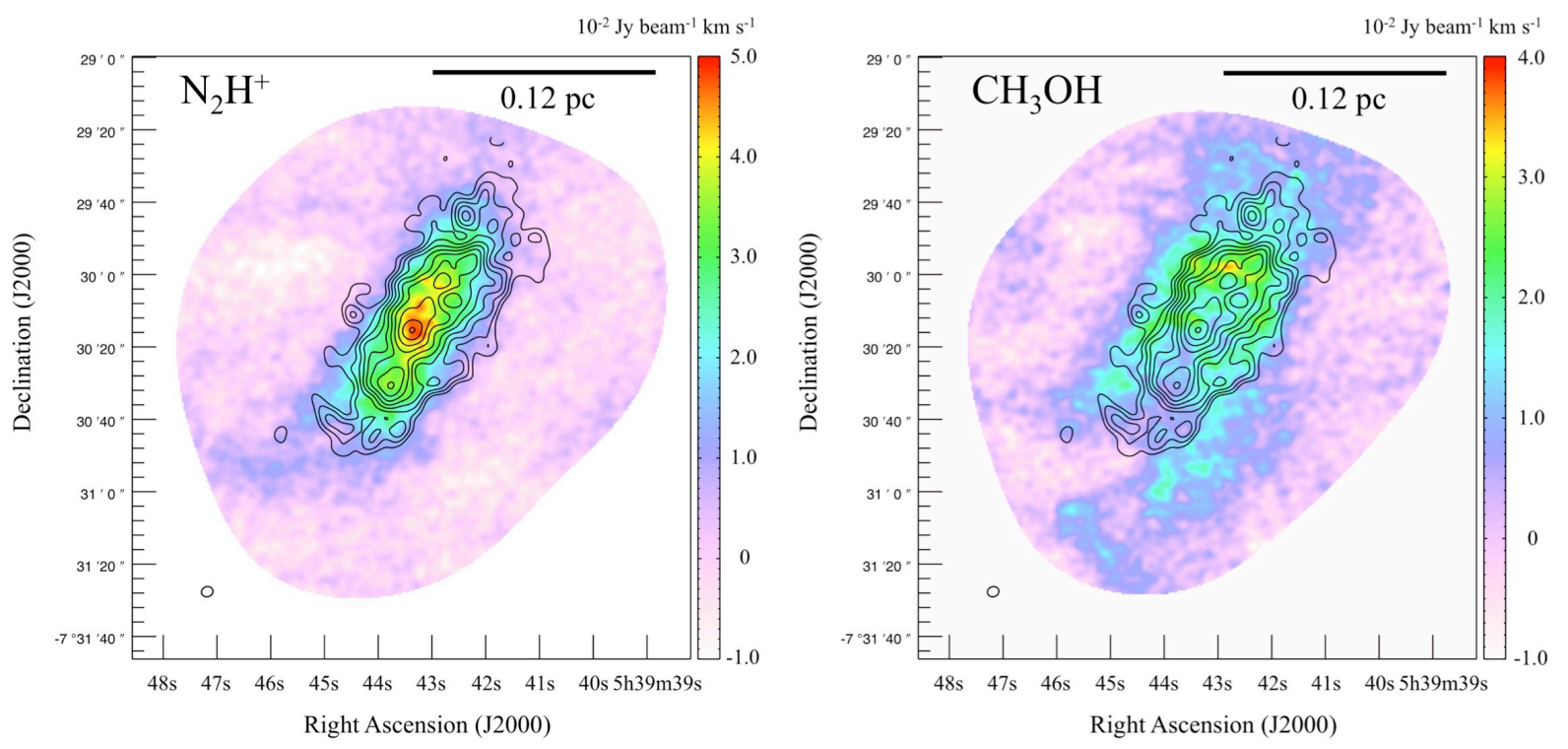

Figure 7. (Right): The $\mathrm{N}_{2} \mathrm{H}^{+}$velocity-integrated intensity image toward TUKH122 core obtained form ALMA-ACA combined data. The velocity range is $3.4-4.0 \mathrm{~km} \mathrm{~s}^{-1}$. The contours represent the $3 \mathrm{~mm}$ dust continuum map and are the same as Figure 4 . The bottom-left circle represents the beamsize of $3 \prime^{\prime \prime} 2 \times 2^{\prime \prime} .9\left(\mathrm{PA}=-73^{\circ}\right)(\mathrm{Left})$ : The $\mathrm{CH}_{3} \mathrm{OH}$ velocity-integrated intensity image toward TUKH122 core obtained form ALMA-ACA combined data. The velocity range is $3.4-4.0 \mathrm{~km} \mathrm{~s}^{-1}$. The contours represent the $3 \mathrm{~mm}$ dust continuum map and are the same as Figure 4. The bottom-left circle represents the beamsize of 3 !' $^{\prime} \times 2{ }^{\prime \prime} .8\left(\mathrm{PA}=-72^{\circ}\right)$.

interesting trend that the northern condensations (MM1, MM2, and MM3) have $0.1-0.2 M_{\odot}$, while the southern condensations (MM4 and MM5) have $\sim 0.4 M_{\odot}$. We discuss the different mass distribution along the filament in the following section.

The total mass of TUKH122 is derived to be $8.4 M_{\odot}$ (2.5 $M_{\odot}$ for ALMA 12-m data) using the ALMA-ACA combined data. Taking into account the total mass of TUKH122 $\left(\sim 29 M_{\odot}\right)$ estimated by Herschel, there is still a large fraction of flux that is not recovered by ALMA. In order to check how the condensation masses vary, we combine our ALMA and ACA data with the Herschel $250 \mu \mathrm{m}$ data using feather in CASA. Note that we rescale the $250 \mu \mathrm{m}$ flux to the $3 \mathrm{~mm}$ flux assuming the dust temperature of $12 \mathrm{~K}$ and ice-covered silicategraphite conglomerate grains with a dust emissivity index of $\beta=2$ modeled by Ormel et al. (2007). We confirm that the total mass is recovered to be $\sim 29 M_{\odot}$. Then, we apply the dendrogram method to the $3 \mathrm{~mm}$ continuum image in the same way as the above. We find that the condensation mass ranges from 0.1 to $0.7 M_{\odot}$. The MM1 to MM4 condensations increase in mass only $\sim 10 \%$ but the MM5 increases $\sim 60 \%$ in mass, suggesting that the condensation masses do not change even when we add the total power except for MM5. However, we should also note that if condensations have relatively extended structures as MM5 does, the masses may be underestimated due to the missing flux.

We also study the dynamical stability of these condensations. Assuming a uniform density structure, the virial mass can be estimated as

$$
M_{\mathrm{vir}}=210 \times\left(\frac{r}{\mathrm{pc}}\right) \times\left(\frac{\Delta v}{\mathrm{~km} \mathrm{~s}^{-1}}\right)^{2} M_{\odot},
$$

where $r$ is the radius and $\Delta v$ is the linewidth (MacLaren et al. 1988). The linewidth is derived by gaussian fitting of the $\mathrm{CH}_{3} \mathrm{OH}\left(J_{K}=2_{0}-1_{0} A^{+}\right)$emission because this line is optically thin. The centroid velocity, linewidth, and virial mass are shown in Table 2. As shown in the table, the condensation mass is much higher than the virial mass. Therefore, these condensations are not in 
virial equilibrium and may collapse immediately unless the magnetic field counterbalances gravity.

\subsection{The molecular line emission of $\mathrm{N}_{2} \mathrm{H}^{+}$and $\mathrm{CH}_{3} \mathrm{OH}$}

Figure 7 shows in colors the velocity-integrated intensity maps of $\mathrm{N}_{2} \mathrm{H}^{+}\left(J=1-0, F_{1}=1-1, F=0-1\right)$ and $\mathrm{CH}_{3} \mathrm{OH}\left(J_{K}=2_{0}-1_{0} A^{+}\right)$emission without the primary beam corrections. The ALMA 12-m and ACA 7 -m data are combined. The $F_{1}=1-1, F=0-1$ transition line is the weakest hyperfine component, and hence, it's optical depth is the smallest. The contours are the 3 mm dust continuum (same as Figure 4). The $3 \mathrm{~mm}$ dust continuum and the $\mathrm{N}_{2} \mathrm{H}^{+}$transition maps show that the intensities concentrate at the central part and have the same peak position. On the other hand, the $\mathrm{CH}_{3} \mathrm{OH}$ emission is weak at the central part of the TUKH122 core, resembling a shell-like structure that surrounds the continuum and $\mathrm{N}_{2} \mathrm{H}^{+}$emission. These configurations are quite similar to the starless cores of L1498 and L1517B, and L1544 (Tafalla et al. 2006; Vastel et al. 2014; Spezzano et al. 2016), indicating that the $\mathrm{CH}_{3} \mathrm{OH}$ and $\mathrm{N}_{2} \mathrm{H}^{+}$ molecules have chemical differentiation.

The $\mathrm{CH}_{3} \mathrm{OH}$ molecule is suggested to be formed on the surface of dust grains via successive hydrogenation of CO (e.g., Watanabe \& Kouchi 2002) and is released by external heating and/or sputtering due to star formation activity. Therefore, the $\mathrm{CH}_{3} \mathrm{OH}$ lines are often used as a tracer of star formation activity and shocks. However, even in the cold quiescent clouds including this object, the $\mathrm{CH}_{3} \mathrm{OH}$ lines have also been observed with moderate intensities. Vastel et al. (2014) suggested that the non-thermal desorption mechanism is important for the observed emission of $\mathrm{CH}_{3} \mathrm{OH}$. Soma et al. (2015) also suggested that the $\mathrm{CH}_{3} \mathrm{OH}$ formed on dust grains is liberated into the gas phase by non-thermal desorption such as photoevaporation caused by cosmic-ray induced UV radiation. According to this mechanism, the column density of gaseous $\mathrm{CH}_{3} \mathrm{OH}$ is proportional to the path length of emitting region along the line of sight. Because the UV radiation is shielded in the core interiors, the emitting region has a shell-like structure. The extended $\mathrm{CH}_{3} \mathrm{OH}$ distribution seems to be consistent with this scenario. Note that the $\mathrm{CH}_{3} \mathrm{OH}$ shell structure is mainly observed in the north region and absent in the southeast. This may be because the $\mathrm{CH}_{3} \mathrm{OH}$ emission is filtered out in the interferometric data if the $\mathrm{CH}_{3} \mathrm{OH}$ emission is widely distributed or the $\mathrm{CO}$ molecule (the precursor of the $\mathrm{CH}_{3} \mathrm{OH}$ ) is less abundant on dust grains in the south region if the southern part is chemically young ( $\mathrm{CO}$ has not been formed yet).

Tatematsu et al. (2014b) also observed TUKH122 core with the $\mathrm{NH}_{3}$ and CCS molecules using VLA and found that the CCS emission surrounds the $\mathrm{NH}_{3}$ core. Figure 8 shows the velocity-integrated intensity map of the ALMA $\mathrm{CH}_{3} \mathrm{OH}$ observations overlaid with the contours of the VLA CCS observations. In comparison with these distributions, we find that the CCS emission is located on the $\mathrm{CH}_{3} \mathrm{OH}$ shell structure or slightly outside the shell.

\subsubsection{Analysis of $\mathrm{N}_{2} \mathrm{H}^{+}$spectra}

Because $\mathrm{N}_{2} \mathrm{H}^{+}$has hyperfine structure, we derive the optical depth $(\tau)$, LSR velocity $\left(V_{\mathrm{LSR}}\right)$, linewidth $(d v)$, and excitation temperature $\left(T_{\text {ex }}\right)$ assuming a uniform excitation temperature in the same way as Ohashi et al.

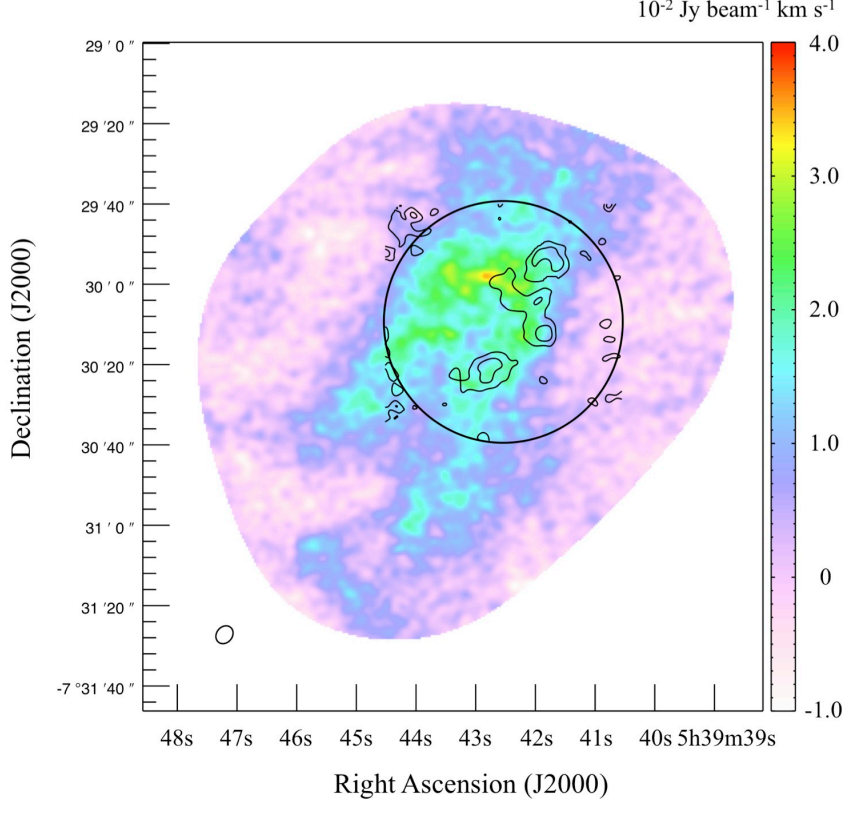

Figure 8. The ALMA $\mathrm{CH}_{3} \mathrm{OH}$ integrated intensity map (color) superimposed on the VLA CCS integrated intensity map (contours). The large circle delineates the primary beam size of the VLA interferometric observations. The contour interval is $6.9 \mathrm{mJy}$ beam ${ }^{-1} \mathrm{~km} \mathrm{~s}^{-1}$, which corresponds to $3 \sigma$ and $1.5 \sigma$ at the map center and at the edge of the primary beam, respectively. In the lower left corner, the synthesized beam of $4 .^{\prime \prime} 7 \times 3$.' $^{\prime \prime}\left(\mathrm{PA}=-40^{\circ}\right)$ for CCS is shown.

(2014, 2016b) using IDL fitting code. However, we were not able to estimate the optical depth values toward the dust peaks because $\mathrm{N}_{2} \mathrm{H}^{+}$line profiles show some features of high optical depths. Such extreme optical depths have been also observed in infrared dark clouds (IRDC) (e.g., Sanhueza et al. 2013). Figure 9 shows an example of $\mathrm{N}_{2} \mathrm{H}^{+}$profile of one pixel spectra obtained by the ALMA-ACA combined image at the dust peak position. The intrinsic intensity ratios of each hyperfine component are represented by the red segments at the bottom of Figure 9 in the optically thin limit (Tiné et al. 2000). The observed intensity-profile does not follow the intrinsic intensity-profile, indicating that most of the hyperfine intensities are saturated. Therefore, we only use the weakest and the most optically thin component $\left(J=1-0, F_{1}=1-1, F=0-1\right)$ to derive physical parameters. The excitation temperature $T_{\text {ex }}$ is assumed to be the maximum intensities of the main component adding the cosmic microwave background of $2.7 \mathrm{~K}$ in each position, ranging from 4 to $6 \mathrm{~K}$. Then we derive the optical depth by using the radiative transfer formula assuming the filling factor of unity. We also derive LSR velocity, and linewidth by gaussian fitting of the optically thinnest line. The fitting is performed for the pixels above $5 \sigma$. We should note that the $3 \mathrm{~mm}$ dust emission is optically thin $\left(\tau_{\text {dust }}<0.1\right)$ and would not affect the line profile.

Figure 10 shows the color maps of these parameters with the $3 \mathrm{~mm}$ dust continuum contours (from $3 \sigma$ with $2 \sigma$ steps). The color map of optical depth indicates that even the weakest $\mathrm{N}_{2} \mathrm{H}^{+}$hyperfine line is moderately optically thick $(\tau \gtrsim 1)$ toward the condensations. In particular, we were not able to calculate the optical depth due to the saturation of the lines in the southern dust lo- 

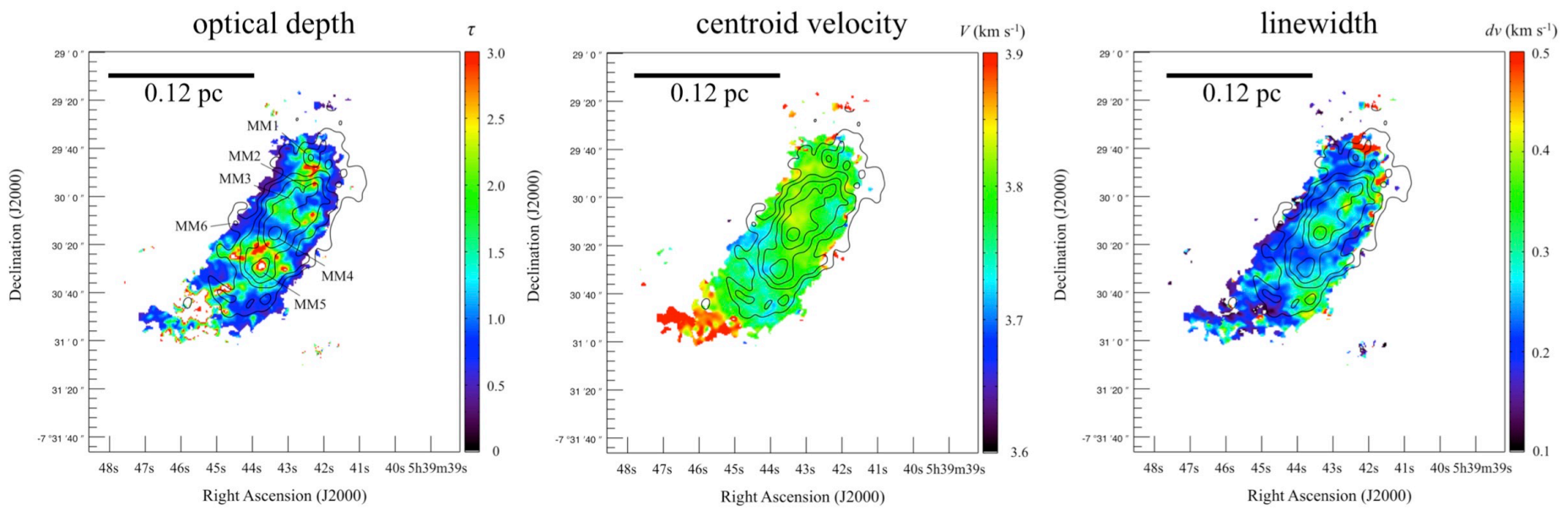

Figure 10. The color maps of the optical depth $(\tau)$, LSR velocity $\left(V_{\mathrm{LSR}}\right)$, and linewidth $(d v)$ of $\mathrm{N}_{2} \mathrm{H}^{+} J=1-0, F_{1}=1-1, F=0-1$ emission derived from by Gaussian fitting of the weakest hyperfine component. The contours are the 3 mm dust continuum emission from $3 \sigma$ with $2 \sigma$ steps. The fitting is performed for the pixels above $5 \sigma$. The optical depth is derived by assuming that the maximum intensity of $\mathrm{N}_{2} \mathrm{H}^{+} J=1-0$ corresponds to the excitation temperature.

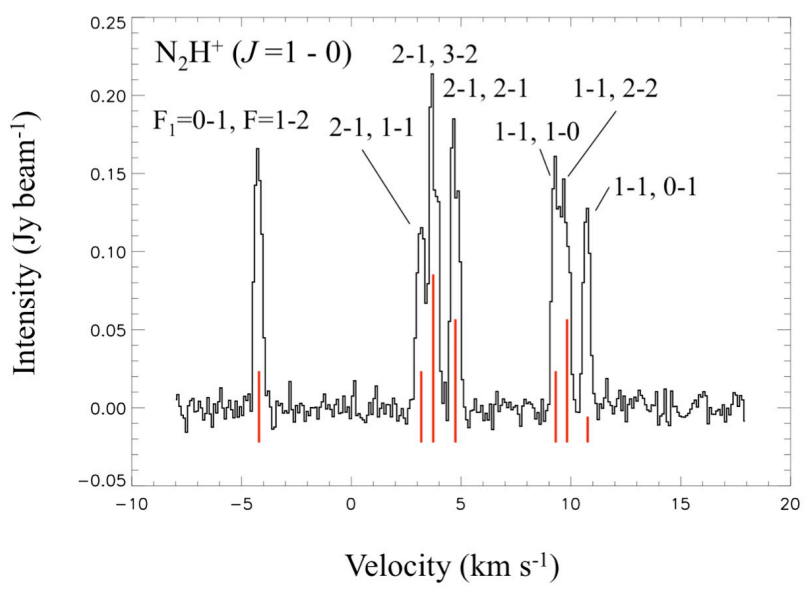

Figure 9. $\mathrm{N}_{2} \mathrm{H}^{+}(J=1-0)$ profile at the MM4 peak position. The velocity resolution is $0.098 \mathrm{kms}^{-1}$.

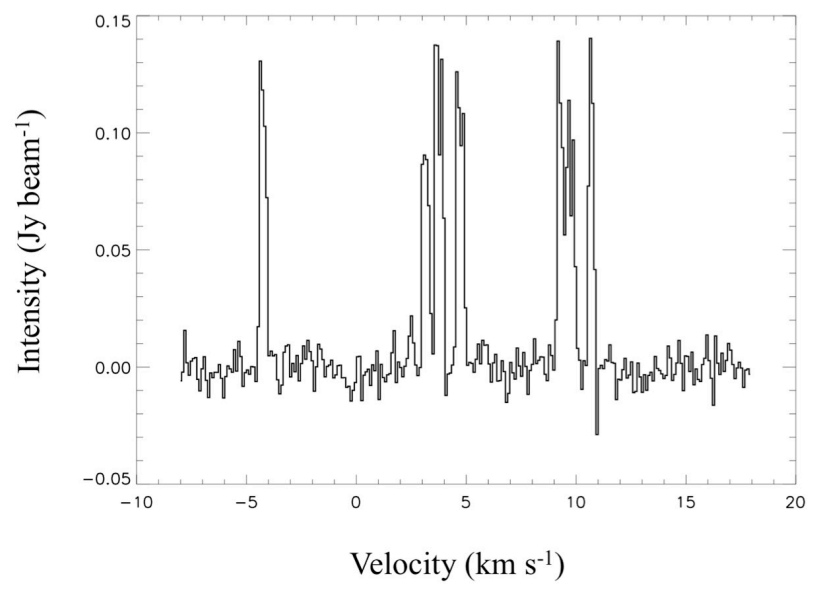

Figure 11. The same as Figure 9 but at the MM5 position. cal peak (MM5). Figure 11 shows the profile toward the MM5 peak position. This profile shows that the weakest hyperfine has the strongest intensity peak. Furthermore, we find two velocity components in the main hyperfine emission of $F_{1}=2-1, F=3-2$. Figure 12 shows zoom up profiles in the same position, MM5. The black line represents the $F_{1}=2-2, F=3-2$ emission at a rest frequency of $93.173777 \mathrm{GHz}$ and the green line represents the $F_{1}=1-1, F=0-1$ emission at a rest frequency of $93.171621 \mathrm{GHz}$. The black line shows a "dip" in the emission profile at $3.7 \mathrm{~km} \mathrm{~s}^{-1}$, while the green line reachs a peak in this gap. Thus, the double peak feature seen in the strongest hyperfine component would be caused by self-absorption effect. In a similar case, a self-absorption in $\mathrm{NH}_{3}$ was found toward the IRDC G028.23-00.19, prestellar clump (Sanhueza et al. 2017). The self-absorption of $\mathrm{N}_{2} \mathrm{H}^{+}$and $\mathrm{NH}_{3}$ lines suggests that the inner part region has higher density and higher excitation temperature than surrounding $\mathrm{N}_{2} \mathrm{H}^{+}$ or $\mathrm{NH}_{3}$ emission regions.

We also find that the optical depth is not correlated well with the dust continuum emission. The MM4 is the peak position in the dust continuum but the optical depth seems to be lower than that in the surrounding region, which may suggest the lower ionization degree in the dense part of the core or the depletion of $\mathrm{N}_{2} \mathrm{H}^{+}$at the densest parts. The N-bearing molecules of $\mathrm{N}_{2} \mathrm{H}^{+}$and $\mathrm{NH}_{3}$ are suggested to be depleted at a density of $\gtrsim 10^{6}$ $\mathrm{cm}^{-3}$ (e.g., Bergin et al. 2002; Pagani et al. 2005).

In the color map of $V_{\mathrm{LSR}}$, there is no evident velocity variation along the filament. Systematic velocity fluctuations is also not found along the ridge of the continuum image. This could be due to the impossibility of tracing velocity fields using optically thick lines (most regions have $\tau \gtrsim 1)$ or the insufficient velocity resolution $(0.098$ $\mathrm{km} \mathrm{s}^{-1}$ ) of our observation to identify infall or fragment motions. On the other hand, in the linewidth map, we find that the linewidth increases toward the condensations. Because the central condensation does not have a high optical depth $(\tau \sim 1)$ in comparison with the surrounding part, broad linewidth is not due to the sat- 


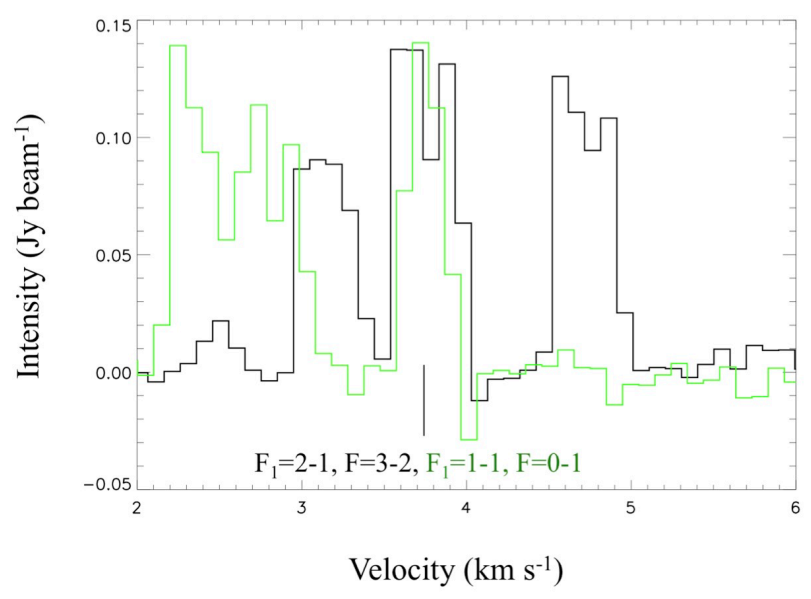

Figure 12. The black line represents $\mathrm{N}_{2} \mathrm{H}^{+} F_{1}=2-1, F=3-2$ profile at the MM5 position with the rest frequency of 93.173777 $\mathrm{GHz}$ and the green line represent the $F_{1}=1-1, F=0-1$ profile with the rest frequency of $93.171621 \mathrm{GHz}$.

uration effect of the line but indicate motions (infall, expansion, or turbulent) toward the condensation. Considering that the $\mathrm{N}_{2} \mathrm{H}^{+}$molecule is partially depleted and the linewidth becomes broader toward the MM4, this condensation may be beginning to collapse. However, our current velocity resolution and the molecular lines are not enough to resolve and investigate the motions in details. It is highly needed to investigate the nature of the enhanced linewidth, i.e, the presence of blue-skewed profile suggesting infall, with higher spectral resolution.

Even though $\mathrm{N}_{2} \mathrm{H}^{+}$is highly optically thick, we roughly estimate the $\mathrm{N}_{2} \mathrm{H}^{+}$abundance at the dust peak position. In the dust peak, we assume the excitation temperature of $T_{\mathrm{ex}}=5.8 \mathrm{~K}$ from the maximum intensity. The optical depth of the $F_{1}=1-1, F=0-1$ line is derived to be $\tau=0.9 \pm 0.1$. Then, the column density can be calculated by following Sanhueza et al. (2012); Mangum \& Shirley (2015). We derive $N_{\mathrm{N}_{2} \mathrm{H}^{+}}=(3.4 \pm 0.4) \times 10^{13} \mathrm{~cm}^{-2}$. On the other hand, the $3 \mathrm{~mm}$ dust continuum shows $N\left(\mathrm{H}_{2}\right)=9.9 \times 10^{22}$ $\mathrm{cm}^{-2}$. Therefore, the $\mathrm{N}_{2} \mathrm{H}^{+}$abundance is derived to be $X\left(\mathrm{~N}_{2} \mathrm{H}^{+}\right)=(3.4 \pm 0.4) \times 10^{-10}$, which is similar to other low-mass star forming regions (e.g., Caselli et al. 2002; Tafalla et al. 2002, 2006; Keto et al. 2004; Friesen et al. 2010)

\subsubsection{Analysis of $\mathrm{CH}_{3} \mathrm{OH}$ spectra}

The $\mathrm{CH}_{3} \mathrm{OH}$ transitions observed are $\left(2_{-1}-1_{-1} E\right)$, $\left(2_{0}-1_{0} A^{+}\right)$, and $\left(2_{0}-1_{0} E\right)$. Assuming LTE and optically thin conditions, we can estimate the column density $\left(N_{\mathrm{CH}_{3} \mathrm{OH}}\right)$ and rotation temperature $\left(T_{\text {rot }}\right)$ using the rotation diagram technique (e.g., Blake et al. 1987). However, $\mathrm{CH}_{3} \mathrm{OH}\left(2_{0}-1_{0} E\right)$ has a low $\mathrm{S} / \mathrm{N}$ ratio. In order to have a significant detection in all 3 lines, we average the profiles from the whole region inside the $5 \sigma$ contour of the ACA $3 \mathrm{~mm}$ dust continuum image. The averaging region shows the magenta contour in Figure 13. Following the population diagram methods (e.g., Goldsmith \& Langer 1999), we fit the three $\mathrm{CH}_{3} \mathrm{OH}$ lines. We follow the procedure described in Sanhueza et al. (2013), which is optimized for cold gas. The obtained column density and rotation temperature are $N_{\mathrm{CH}_{3} \mathrm{OH}}=(1.1 \pm 0.1) \times 10^{13}$ $\mathrm{cm}^{-2}$ and $T_{\text {rot }}=10.8 \pm 0.4 \mathrm{~K}$. This rotation temperature is consistent with $T_{\text {rot }}=10.6 \mathrm{~K}$ derived by using the $\mathrm{NH}_{3}(J, K=1,1)$ and $(J, K=2,2)$ lines (Ohashi et al. $2016 \mathrm{~b})$. The rotation temperature is the same value even if the different molecular lines are used, suggesting the isothermal core with the LTE condition. The optical depth of the strongest transition $\left(2_{0}-1_{0} A^{+}\right)$ is $\sim 0.1$, and hence, it is optically thin. The average $\mathrm{H}_{2}$ column density is derived to be $N\left(\mathrm{H}_{2}\right)=3.8 \times 10^{22} \mathrm{~cm}^{-2}$ from the $3 \mathrm{~mm}$ dust continuum observations. Therefore, the $\mathrm{CH}_{3} \mathrm{OH}$ abundance is $\mathrm{X}\left(\mathrm{CH}_{3} \mathrm{OH}\right) \sim 2.9 \times 10^{-10}$. Soma et al. (2015) estimated the abundance of $\mathrm{CH}_{3} \mathrm{OH}$ to be $\sim 10^{-9}$ toward the cold quiescent core, TMC1 (CP). Tafalla et al. (2006) also estimated the abundance to be $X\left(\mathrm{CH}_{3} \mathrm{OH}\right) \sim 6 \times 10^{-10}$ in the L1498 and L1517B prestellar cores. TUKH122 has similar $\mathrm{CH}_{3} \mathrm{OH}$ abundance to these prestellar cores. In particular, the $\mathrm{CH}_{3} \mathrm{OH}$ abundance can be even lower at the central part because this value is the average over the whole region. Assuming that the $\mathrm{CH}_{3} \mathrm{OH}\left(2_{0}-1_{0} A^{+}\right)$line is optically thin and $T_{\text {rot }}=10.8 \mathrm{~K}$, we estimate the column density, $N_{\mathrm{CH}_{3} \mathrm{OH}}$, at the dust peak position MM4 by using the rotation temperature derived above. By comparing the velocity-integrated intensities, the column density is derived to be $N_{\mathrm{CH}_{3} \mathrm{OH}}=1.3 \times 10^{13} \mathrm{~cm}^{-2}$ at this dust peak position, and then the $\mathrm{CH}_{3} \mathrm{OH}$ abundance is $X\left(\mathrm{CH}_{3} \mathrm{OH}\right) \sim 1.3 \times 10^{-10}$.

Figure 13 shows in color maps of the peak intensity, $V_{\mathrm{LSR}}$, and linewidth of $\mathrm{CH}_{3} \mathrm{OH}$. The peak temperature map clearly shows the shell like structure and the dust condensations are located within this shell. The $V_{\text {LSR }}$ map shows some systematic variations within the core. The northern edge is slightly red-shifted in velocity, while the southern edge is blue-shifted, suggesting core rotation or gas inflow motions along the filamentary structure. Furthermore, the south-eastern side is blue-shifted velocity, while the shouth-western side is red shifted. The velocity structure is complicated but the velocity difference might indicate the contraction or twisted motions of the core.

The linewdith map shows no systemic variations along the filament. However, the dust peak position (MM4) has a broad linewidth of $\Delta v \sim 0.4 \mathrm{~km} \mathrm{~s}^{-1}$, which is the same tendency with that in the $\mathrm{N}_{2} \mathrm{H}^{+}$. These broad linewidths would thus suggest infall motions. Interestingly, the linewidth color maps of $\mathrm{N}_{2} \mathrm{H}^{+}$and $\mathrm{CH}_{3} \mathrm{OH}$ show a small value of $d v \lesssim 0.2 \mathrm{~km} \mathrm{~s}^{-1}$ at the edge of the condensations and our velocity resolution marginally resolve the lines. The linewidth of $0.2 \mathrm{~km} \mathrm{~s}^{-1}$ is close to the thermal motion because thermal linewidth corresponds to be $0.14 \mathrm{~km} \mathrm{~s}^{-1}$ for $\mathrm{N}_{2} \mathrm{H}^{+}$and $0.13 \mathrm{~km} \mathrm{~s}^{-1}$ for $\mathrm{CH}_{3} \mathrm{OH}$ at $12 \mathrm{~K}$. The narrow linewith of the core edge indicates that the turbulence is almost dissipated in the filament, and the non-thermal motions within the core could indicate infall motions or some systemic velocity variations such as rotation and fragmentation.

The important difference between TUKH122 and the other prestellar cores in nearby clouds is that TUKH122 consists of at least three dense condensations within the one $\mathrm{CH}_{3} \mathrm{OH}$ shell. The TUKH122 core is more massive than low-mass dense cores $\left(\sim M_{\odot}\right)$ (e.g., Onishi et al. 2002; Alves et al. 2007) even though the size is comparable, implying that the TUKH122 core is denser. The 

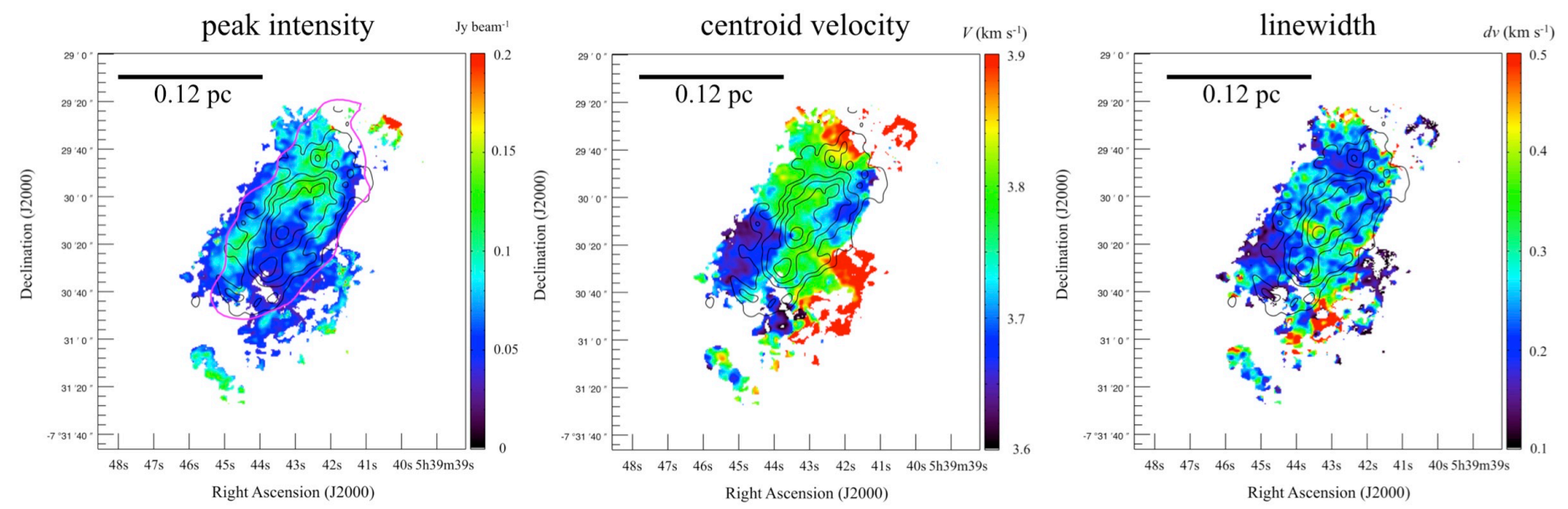

Figure 13. The color maps of the peak intensity $\left(\mathrm{Jy}_{\text {beam }}{ }^{-1}\right)$, LSR velocity $\left(V_{\mathrm{LSR}}\right)$, and linewidth $(d v)$ of $\mathrm{CH}_{3} \mathrm{OH}\left(J=2_{0,2}-1_{0,1} \mathrm{~A}^{+}\right)$ emission derived by the Gaussian fitting. The fitting is performed for the pixels above $5 \sigma$ of the $\mathrm{CH}_{3} \mathrm{OH}$ emission. Black contours show the ALMA-ACA $3 \mathrm{~mm}$ dust continuum emission from $3 \sigma$ with $2 \sigma$ steps. The magenta contour shows the $5 \sigma$ contour of the ACA 3 mm dust image for averaging the $\mathrm{CH}_{3} \mathrm{OH}$ profile.

denser core would enhance $\mathrm{CH}_{3} \mathrm{OH}$ shell structure because UV radiation is only irradiated on the outer layer of the core.

\section{DISCUSSION}

\subsection{Density profile}

To investigate the formation of the condensations, we study the density structure along the filament. Figure 14 shows the column density profiles taken from the maximum position along the filament direction (see also Figure 5). The reference position here is the MM4 condensation corresponding to the peak flux in the ALMAACA observations. The Herschel single dish observations indicate almost flat density structure due to the large beamsize. On the other hand, the ALMA-ACA observations show column density variations. The green line represents a sinusoidal column-density variation with an interval of $0.035 \mathrm{pc}$ to visually match the observed variations. The southern part (MM3, 4, and 5) seems to be nicely fitted by this sinusoidal variation.

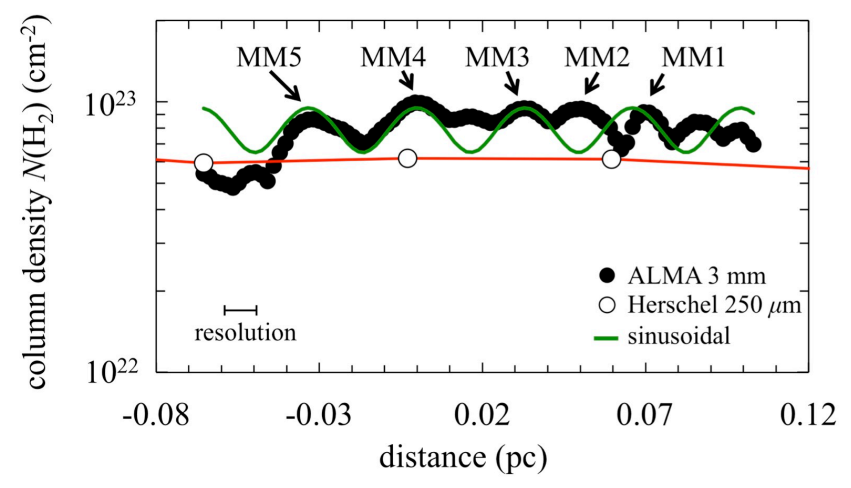

Figure 14. The maximum column density profile along the elongated core taken by ALMA-ACA observations and the Herschel $250 \mu \mathrm{m}$ observations. The spatial resolution is $\sim 0.01 \mathrm{pc}$. The green line represents a sinusoidal motion with an interval of 0.035 pc.

The interval of $0.035 \mathrm{pc}$ is consistent with the sepa- rations between neighbor sources in the OMC-2 and 3 regions (Takahashi et al. 2013; Kainulainen et al. 2017). In OMC-1, another part of the Orion A cloud, a separation of $\sim 0.01 \mathrm{pc}$ has been reported by Teixeira et al. (2016); Palau et al. (2017). Since the OMC-1 is the most massive part in the Orion A cloud, the fragmentation might occur at denser region and the separations would then become shorter. The separations and fragmentation processes have also been studied in other regions including high mass star forming regions and suggested to be governed by thermal Jeans processes (e.g., Kainulainen et al. 2013; Beuther et al. 2015; Palau et al. 2015; Busquet et al. 2016). Assuming that the fragmentation occurs by the Jeans instability, we can estimate the separation of the fragment at this region. With the assumption of an infinite size and a uniform density, the Jeans length is described as follows (Jeans 1902):

$$
\lambda_{\text {Jeans }}=\sqrt{\frac{\pi c_{s}^{2}}{G \rho_{0}}},
$$

where $G$ is the gravitational constant, $\rho_{0}$ is the mean density, and $c_{s}$ is the sound speed of $0.2 \mathrm{~km} \mathrm{~s}^{-1}$ at $12 \mathrm{~K}$. Takahashi et al. (2013) also analyzed the Jeans length in the case of an infinitely long static and cylindrical isothermal cloud following Nakamura et al. (1993); Wiseman \& Ho (1998) and described as

$$
\lambda_{\text {Jeans }} \sim \frac{20 c_{s}}{\sqrt{4 \pi G \rho_{0}}} .
$$

From the column density profile of the ALMA-ACA observations in Figure 6, we derive the central density, $\rho_{c} \sim 5.5 \times 10^{5} \mathrm{~cm}^{-3}$ assuming the numerical factor of $A=\pi / 2$. The Jeans length is derived to be $\sim 0.031 \mathrm{pc}$ and $\sim 0.099 \mathrm{pc}$, respectively following the equations of (5) and (6) assuming the temperature of $12 \mathrm{~K}$. The thermal Jeans instability with the assumption of an infinite and a uniform density, the equation (5), would be better explained to form the condensations. Therefore, the fragmentation occurs within the elongated core having a density of $\sim 10^{5} \mathrm{~cm}^{-3}$ and the denser condensations 
with a density of $\sim 10^{6-7} \mathrm{~cm}^{-3}$ are formed by the thermal Jeans instability. Note that assuming $\lambda_{\text {Jeans }}=0.035$ $\mathrm{pc}$, the density is derived to be $n\left(\mathrm{H}_{2}\right) \sim 4.4 \times 10^{5} \mathrm{~cm}^{-3}$ from the equation (5).

The northern part (MM1 and 2) seems to have a shorter interval of $\sim 0.02 \mathrm{pc}$. An inclination might cause a shorter interval even if these condensations have the same interval of $\sim 0.035 \mathrm{pc}$ or the Jeans instability at higher densities may occur. We should also note that theses northern condensations are located on the $\mathrm{CH}_{3} \mathrm{OH}$ shell structure, suggesting the edge of the TUKH122 core. Therefore, the edge instabilities may also affect the separation of the condensations (Pon et al. 2011, 2012). The lower mass condensations with shorter intervals may be formed in the edge region.

We also discuss a possibility whether the condensations will merge or not. From the $\mathrm{CH}_{3} \mathrm{OH}$ observations, the relative velocity of the surrounding condensation for MM 4 are $0.02-0.05 \mathrm{~km} \mathrm{~s}^{-1}$. To merge the condensations, we need at least $4 \times 10^{5} \mathrm{yr}$ assuming the separation of 0.02 pc and velocity of $0.05 \mathrm{~km} \mathrm{~s}^{-1}$. On the other hand, the free fall time at a density of $10^{6} \mathrm{~cm}^{-3}$ will be $4 \times 10^{4} \mathrm{yr}$. Therefore, the star formation can be started before these condensations merge. It is unlikely that these condensations merge to a larger scale. However, the magnetic field may be supporting the condensations and makes the star formation time scale longer (e.g., Crutcher et al. 2004; Ward-Thompson et al. 2007). It is suggested that the ambipolar diffusion timescale is about 10 times longer than the free fall time (Nakano 1998). In this case, the merging might be possible.

\subsection{Possibility of multiple star formation}

In the previous section, we identified the 6 condensations with a mass of $\sim 0.1-0.4 M_{\odot}$ in the core and they are gravitationally bound. However, the total mass of the condensations is only $1.2 M_{\odot}$ and a large portion of the mass is located outside of them. One possibility of the small mass fraction of the condensations is that we may only detect the peak region of the condensations, and the condensations may have larger radius and mass. For example, if the condensations have unstable Bonnor-Ebert sphere, they have a large contrast in density between the center and outer part. The condensations might be able to identify only the center part due to our spatial resolutions and sensitivities. Actually, the size of the condensations is derived to be $r \sim 0.006$ $\mathrm{pc}$ even though we find the separation of $0.02-0.035 \mathrm{pc}$ between them. If we use the separations as the size of these condensations, the mass is derived to be $0.3-1$ $M_{\odot}$. Even if the condensations have masses of $0.3-1$ $M_{\odot}$, they only have a small amount of the mass and the virial parameters are still lower than unity. These condensations may collapse immediately. Another possibility is that very low-mass stars $\left(\sim 0.03-0.1 M_{\odot}\right)$ are formed in these condensations in this region. In either case, the multiple star system may be formed in the core along the filament. We suggest that the fragmentation is still important in the dense core to form multiple stars.

\section{CONCLUSIONS}

We have presented the ALMA high spatial resolution observations of $3 \mathrm{~mm}$ dust continuum, the hyperfinecomponents lines of $\mathrm{N}_{2} \mathrm{H}^{+}(J=1-0)$, and the $\mathrm{CH}_{3} \mathrm{OH}$
$\left(J_{K}=2_{K}-1_{K}\right)$ lines toward the TUKH122 core. This core is likely on the verge of star formation because the turbulence is almost completely dissipated and is a chemically evolved core among starless core in the Orion A cloud. Our main results are summarized as follows.

1. The column density profile perpendicular to the parent filamentary structure including the elongated core is similar to the Plummer-like function. The Herschel observations show the power law of $p=1.6$, while the ALMA-ACA observations shows a power law of $p=4.0$. This difference will be explained in terms of the missing flux of the interferometric observations. The condensations are embedded in the parent elongated core.

2. ALMA 12-m dust continuum emission shows compact dense condensations, while ACA 7-m image shows the oval structure due to the larger beamsize and recovering the extended emission. By combining these data and applying the dendrogram method, we find condensations within the core with a mass range of $0.1-0.4$ $M_{\odot}$ and a deconvolved radius of $0.003-0.01$ pc along the filament. The densities are $n \sim 10^{6-7} \mathrm{~cm}^{-3}$, which are an order of magnitude higher than typical low-mass prestellar cores.

3. We find different distributions between $\mathrm{N}_{2} \mathrm{H}^{+}$and $\mathrm{CH}_{3} \mathrm{OH}$ emission. The $\mathrm{N}_{2} \mathrm{H}^{+}$distribution is similar to the dust continuum but seems to be frozen-out in the dust peak MM4, while the $\mathrm{CH}_{3} \mathrm{OH}$ distribution shows a shell-like structure. The extended $\mathrm{CH}_{3} \mathrm{OH}$ distribution suggests that the $\mathrm{CH}_{3} \mathrm{OH}$ molecule formed on dust grain is released into gas phase by non-thermal desorption such as desorption caused by cosmic-ray induced UV radiation. We also find the absorption feature in the $\mathrm{N}_{2} \mathrm{H}^{+} F_{1}=2-1 F=3-2$ component toward the condensation, MM5, due to the self-absorption effect. Even though $\mathrm{N}_{2} \mathrm{H}^{+}$has been used as an optically thin dense gas tracer, we show that this molecule becomes optically thick in this core.

4. The separations of these condensations are $\sim 0.035$ pc in the southern part, which is similar to that in the OMC- $2 / 3$ region, the active star forming region in the Orion A cloud. The Jeans length corresponds to be $n\left(\mathrm{H}_{2}\right) \sim 4.4 \times 10^{5} \mathrm{~cm}^{-3}$, which is almost consistent with that of the central density of the core. Therefore the fragmentation still occurs in the prestellar core by thermal Jeans instability at a density of $\sim 10^{5} \mathrm{~cm}^{-3}$ and the denser condensations with a density of $10^{6-7} \mathrm{~cm}^{-3}$ are formed. In the northern part corresponding to the $\mathrm{CH}_{3} \mathrm{OH}$ shell region, we find that the condensations are less massive with shorter intervals, which may imply that the edge instabilities affect the fragmentation.

5. The condensations are not in virial equilibrium and may collapse immediately unless the magnetic field counterbalances gravity. However, the total mass of the condensations is a small fraction of the total TUK122 core mass. The condensations might identify only the central part due to our spatial resolutions and sensitivities and they may have masses of $0.3-1 M_{\odot}$. Another possibility is that the very low-mass stars $\left(\sim 0.03-0.1 M_{\odot}\right)$ are formed in the small condensations in this region. In either case, multiple stars may be formed in the core. We suggest that the fragment due to the thermal instability is still important to form a multiple star system. 
We gratefully appreciate the comments from the anonymous referee that significantly improved this article. S.O. also thank Kazuya Saigo for helpful discussions. This paper makes use of the following ALMA data: ADS/JAO.ALMA\#2015.1.01025.S. ALMA is a partnership of ESO (representing its member states), NSF (USA) and NINS (Japan), together with NRC (Canada), NSC and ASIAA (Taiwan), and KASI (Republic of Korea), in cooperation with the Republic of Chile. The Joint ALMA Observatory is operated by ESO, AUI/NRAO and NAOJ.

This research has also made use of data from the Herschel Gould Belt survey (HGBS) project (http://gouldbelt-herschel.cea.fr). The HGBS is a Herschel Key Programme jointly carried out by SPIRE Specialist Astronomy Group 3 (SAG 3), scientists of several institutes in the PACS Consortium (CEA Saclay, INAFIFSI Rome and INAF-Arcetri, KU Leuven, MPIA Heidelberg), and scientists of the Herschel Science Center (HSC).

Data analysis was in part carried out on common use data analysis computer system at the Astronomy Data Center, ADC, of the National Astronomical Observatory of Japan.

Facilities: ALMA, Herschel

\section{REFERENCES}

Aikawa, Y., Ohashi, N., Inutsuka, S.-i., Herbst, E., \& Takakuwa, S. 2001, ApJ, 552, 639

Alves, J. F., Lada, C. J., \& Lada, E. A. 2001, Nature, 409, 159

Alves, J., Lombardi, M., \& Lada, C. J. 2007, A\&A, 462, L17

André, P., Men'shchikov, A., Bontemps, S., et al. 2010, A\&A, 518, L102

André, P., Di Francesco, J., Ward-Thompson, D., et al. 2014, Protostars and Planets VI, 27

Arzoumanian, D., André, P., Didelon, P., et al. 2011, A\&A, 529, L6

Bally, J., Langer, W. D., Stark, A. A., \& Wilson, R. W. 1987, ApJ, 312, L45

Benson, P. J., \& Myers, P. C. 1989, ApJS, 71, 89

Bergin, E. A., Alves, J., Huard, T., \& Lada, C. J. 2002, ApJ, 570 , L101

Beuther, H., Ragan, S. E., Johnston, K., et al. 2015, A\&A, 584, A67

Blake, G. A., Sutton, E. C., Masson, C. R., \& Phillips, T. G. 1987, ApJ, 315, 621

Busquet, G., Estalella, R., Palau, A., et al. 2016, ApJ, 819, 139

Bonnor, W. B. 1956, MNRAS, 116, 351

Caselli, P., Walmsley, C. M., Zucconi, A., et al. 2002, ApJ, 565, 331

Crutcher, R. M., Nutter, D. J., Ward-Thompson, D., \& Kirk, J. M. 2004, ApJ, 600, 279

Da Rio, N., Tan, J. C., Covey, K. R., et al. 2016, ApJ, 818, 59

Dunham, M. M., Offner, S. S. R., Pineda, J. E., et al. 2016, ApJ, 823,160

Ebert, R. 1955, ZAp, 37, 217

Enoch, M. L., Evans, N. J., II, Sargent, A. I., et al. 2008, ApJ, $684,1240-1259$

Friesen, R. K., Di Francesco, J., Shimajiri, Y., \& Takakuwa, S. 2010, ApJ, 708, 1002

Goldsmith, P. F., \& Langer, W. D. 1999, ApJ, 517, 209

Goldsmith, P. F., Heyer, M., Narayanan, G., et al. 2008, ApJ, $680,428-445$

Goodman, A. A., Rosolowsky, E. W., Borkin, M. A., et al. 2009, Nature, 457,63

Hill, T., Motte, F., Didelon, P., et al. 2011, A\&A, 533, A94

Iguchi, S., Morita, K.-I., Sugimoto, M., et al. 2009, PASJ, 61, 1

Inutsuka, S.-i., \& Miyama, S. M. 1997, ApJ, 480, 681

Jeans, J. H. 1902, Philosophical Transactions of the Royal Society of London Series A, 199, 1
Kainulainen, J., Ragan, S. E., Henning, T., \& Stutz, A. 2013, A\&A, 557, A120

Kainulainen, J., Stutz, A. M., Stanke, T., et al. 2017, A\&A, 600, A141

Kandori, R., Nakajima, Y., Tamura, M., et al. 2005, AJ, 130, 2166

Keto, E., Rybicki, G. B., Bergin, E. A., \& Plume, R. 2004, ApJ, 613,355

Kim, M. K., Hirota, T., Honma, M., et al. 2008, PASJ, 60, 991

Kirk, H., Dunham, M. M., Di Francesco, J., et al. 2017, ApJ, 838, 114

Lombardi, M., Bouy, H., Alves, J., \& Lada, C. J. 2014, A\&A, 566, A 45

Lada, C. J., \& Lada, E. A. 2003, ARA\&A, 41, 57

Matsumoto, T., \& Hanawa, T. 2003, ApJ, 595, 913

McKee, C. F., \& Ostriker, E. C. 2007, ARA\&A, 45, 565

Machida, M. N., Inutsuka, S.-i., \& Matsumoto, T. 2008, ApJ, $676,1088-1108$

MacLaren, I., Richardson, K. M., \& Wolfendale, A. W. 1988, ApJ, 333, 821

Mangum, J. G., \& Shirley, Y. L. 2015, PASP, 127, 266

McMullin, J. P., Waters, B., Schiebel, D., Young, W., \& Golap, K. 2007, Astronomical Data Analysis Software and Systems XVI, 376,127

Megeath, S. T., Gutermuth, R., Muzerolle, J., et al. 2012, AJ, 144,192

Megeath, S. T., Gutermuth, R., Muzerolle, J., et al. 2016, AJ, 151,5

Myers, P. C., \& Benson, P. J. 1983, ApJ, 266, 309

Nakamura, F., Hanawa, T., \& Nakano, T. 1993, PASJ, 45, 551

Nakano, T. 1998, ApJ, 494, 587

Nutter, D., Kirk, J. M., Stamatellos, D., \& Ward-Thompson, D. 2008, MNRAS, 384, 755

Ormel, C. W., Spaans, M., \& Tielens, A. G. G. M. 2007, A\&A, 461,215

Ostriker, J. 1964, ApJ, 140, 1056

Ohashi, S., Tatematsu, K., Choi, M., et al. 2014, PASJ, 66, 119

Ohashi, S., Tatematsu, K., Fujii, K., et al. 2016a, PASJ, 68, 3

Ohashi, S., Tatematsu, K., Sanhueza, P., et al. 2016b, MNRAS, 459, 4130

Ohashi, S., Sanhueza, P., Chen, H.-R. V., et al. 2016c, ApJ, 833, 209

Onishi, T., Mizuno, A., Kawamura, A., Tachihara, K., \& Fukui, Y. 2002, ApJ, 575, 950

Pagani, L., Pardo, J.-R., Apponi, A. J., Bacmann, A., \& Cabrit, S. 2005, A\&A, 429, 181

Palau, A., Ballesteros-Paredes, J., Vázquez-Semadeni, E., et al. 2015, MNRAS, 453, 3785

Palau, A., Zapata, L. A., Roman-Zuniga, C. G., et al. 2017, arXiv:1706.04623

Palmeirim, P., André, P., Kirk, J., et al. 2013, A\&A, 550, A38

Pineda, J. E., Rosolowsky, E. W., \& Goodman, A. A. 2009, ApJ, 699, L134

Pineda, J. E., Goodman, A. A., Arce, H. G., et al. 2010, ApJ, $712, \mathrm{~L} 116$

Pineda, J. E., Goodman, A. A., Arce, H. G., et al. 2011, ApJ, 739, L2

Pineda, J. E., Offner, S. S. R., Parker, R. J., et al. 2015, Nature, 518, 213

Polychroni, D., Schisano, E., Elia, D., et al. 2013, ApJ, 777, L33

Pon, A., Johnstone, D., \& Heitsch, F. 2011, ApJ, 740, 88

Pon, A., Toalá, J. A., Johnstone, D., et al. 2012, ApJ, 756, 145

Roy, A., Martin, P. G., Polychroni, D., et al. 2013, ApJ, 763, 55

Rosolowsky, E. W., Pineda, J. E., Kauffmann, J., \& Goodman, A. A. 2008, ApJ, 679, 1338-1351

Sanhueza, P., Jackson, J. M., Foster, J. B., et al. 2012, ApJ, 756, 60

Sanhueza, P., Jackson, J. M., Foster, J. B., et al. 2013, ApJ, 773, 123

Sanhueza, P., Jackson, J. M., Zhang, Q., et al. 2017, ApJ, 841, 97

Spezzano, S., Bizzocchi, L., Caselli, P., Harju, J., \& Brünken, S. 2016, A\&A, 592, L11

Stodólkiewicz, J. S. 1963, AcA, 13, 30

Suzuki, H. 1983, ApJ, 272, 579

Suzuki, H., Yamamoto, S., Ohishi, M., et al. 1992, ApJ, 392, 551

Soma, T., Sakai, N., Watanabe, Y., \& Yamamoto, S. 2015, ApJ, 802,74 
Tafalla, M., Myers, P. C., Caselli, P., Walmsley, C. M., \& Comito, C. 2002, ApJ, 569, 815

Tafalla, M., Santiago-García, J., Myers, P. C., et al. 2006, A\&A, 455,577

Tatematsu, K., Umemoto, T., Kameya, O., et al. 1993, ApJ, 404, 643

Tatematsu, K., Ohashi, S., Umemoto, T., et al. 2014a, PASJ, 66, 16

Tatematsu, K., Hirota, T., Ohashi, S., et al. 2014b, ApJ, 789, 83

Tatematsu, K., Liu, T., Ohashi, S., et al. 2017, ApJS, 228, 12

Takahashi, S., Ho, P. T. P., Teixeira, P. S., Zapata, L. A., \& Su, Y.-N. 2013, ApJ, 763, 57

Teixeira, P. S., Takahashi, S., Zapata, L. A., \& Ho, P. T. P. 2016, A\&A, 587, A47

Tokuda, K., Onishi, T., Saigo, K., et al. 2014, ApJ, 789, L4
Tokuda, K., Onishi, T., Matsumoto, T., et al. 2016, ApJ, 826, 26 Tiné, S., Roueff, E., Falgarone, E., Gerin, M., \& Pineau des Forêts, G. 2000, A\&A, 356, 1039

Watanabe, N., \& Kouchi, A. 2002, ApJ, 571, L173

Wiseman, J. J., \& Ho, P. T. P. 1998, ApJ, 502, 676

Ward-Thompson, D., Scott, P. F., Hills, R. E., \& Andre, P. 1994, MNRAS, 268, 276

Ward-Thompson, D., André, P., Crutcher, R., et al. 2007, Protostars and Planets V, 33

Whitworth, A. P., \& Ward-Thompson, D. 2001, ApJ, 547, 317

Vastel, C., Ceccarelli, C., Lefloch, B., \& Bachiller, R. 2014, ApJ, 795, L2 